\title{
Enhancing Insurer Value Using Reinsurance and Value-at-Risk Criterion
}

\author{
Ken Seng Tan ${ }^{\mathrm{a}, \mathrm{b}}$ and Chengguo Weng ${ }^{\mathrm{a}}$ \\ ${ }^{a}$ Department of Statistics and Actuarial Science, University of Waterloo, Waterloo, Ontario, \\ N2L 3G1, Canada. \\ E-mail: kstan@uwaterloo.ca \\ ${ }^{\mathrm{b}}$ China Institute for Actuarial Science, Central University of Finance and Economics, Beijing, China. \\ E-mail: c2weng@uwaterloo.ca
}

The quest for optimal reinsurance design has remained an interesting problem among insurers, reinsurers, and academicians. An appropriate use of reinsurance could reduce the underwriting risk of an insurer and thereby enhance its value. This paper complements the existing research on optimal reinsurance by proposing another model for the determination of the optimal reinsurance design. The problem is formulated as a constrained optimization problem with the objective of minimizing the value-at-risk of the net risk of the insurer while subjecting to a profitability constraint. The proposed optimal reinsurance model, therefore, has the advantage of exploiting the classical tradeoff between risk and reward. Under the additional assumptions that the reinsurance premium is determined by the expectation premium principle and the ceded loss function is confined to a class of increasing and convex functions, explicit solutions are derived. Depending on the risk measure's level of confidence, the safety loading for the reinsurance premium, and the expected profit guaranteed for the insurer, we establish conditions for the existence of reinsurance. When it is optimal to cede the insurer's risk, the optimal reinsurance design could be in the form of pure stop-loss reinsurance, quota-share reinsurance, or a combination of stop-loss and quota-share reinsurance.

The Geneva Risk and Insurance Review (2012) 37, 109-140. doi:10.1057/grir.2011.5; published online 23 August 2011

Keywords: value-at-risk (VaR); optimal reinsurance; expectation premium principle; linear programming in infinite dimensional spaces

\section{Introduction}

The importance of sound risk management for financial institutions and insurance enterprises has been dramatically highlighted by the subprime crisis. Risk professionals are constantly seeking better risk measures to quantify risks associated with market, credit, operational, catastrophic, and many others. Risk measures such as value-at-risk (VaR) and conditional VaR or conditional tail expectation (CTE) have been proposed. Among these risk 
measures, VaR has emerged to be the most prominent risk management tool, despite its potential flaw for quantifying risks. Artzner et al. ${ }^{1}$ have shown that $\mathrm{VaR}$ is not a coherent measure of risk because it fails to satisfy the subadditivity property. See also Basak and Shapiro ${ }^{2}$ and Yamai and Yoshiba. ${ }^{3}$ The popularity of $\mathrm{VaR}$ in part is due to its simplicity and in part is driven by the regulatory requirement. ${ }^{4}$ Initial utilization of $\mathrm{VaR}$ is predominantly confined to quantifying market risk exposure for traders, corporate treasurers, and dealers. ${ }^{5}$ Subsequently the use of $\mathrm{VaR}$ (as well as other risk measures such as CTE) as a criterion for optimal portfolio construction has sparked considerable interest among practitioners and researchers. ${ }^{6}$ While these results shed some insights on the optimal construction of portfolios, researchers have also acknowledged the computational difficulties arising from adopting VaR in an optimization model. For example, as pointed out in Gaivoronski and $\mathrm{Pflug}^{7}[t]$ he VaR optimization problem is nonconvex, may exhibit many local minima and is of combinatorial character, i.e. exhibits exponential growth in computational complexity.

In recent years, these risk measures have also been exploited for determining the optimal policy in the context of insurance and reinsurance applications. For example, Wang et al., ${ }^{8}$ Huang, ${ }^{9}$ and Zhou and $\mathrm{Wu}^{10}$ demonstrated that employing VaR as a constraint could assist an insured in determining his or her optimal insurance policy. By minimizing VaR or CTE of the total risk exposure of an insurer, Cai and $\operatorname{Tan}^{11}$ and Cai et al. ${ }^{12}$ derived explicitly the optimal reinsurance treaties for the insurer. ${ }^{13}$ While analytic solutions have been derived in the above reinsurance models, these results can be criticized on the ground that the optimality is based exclusively on minimizing an insurer's risk exposure. In practice, an insurer is concerned not only with its exposure to risk but also its profitability of insuring the underlying risks. To elaborate this point, let us first note that when an insurer uses reinsurance to cede (or transfer) part of its loss to a reinsurer, the insurer is liable to pay reinsurance

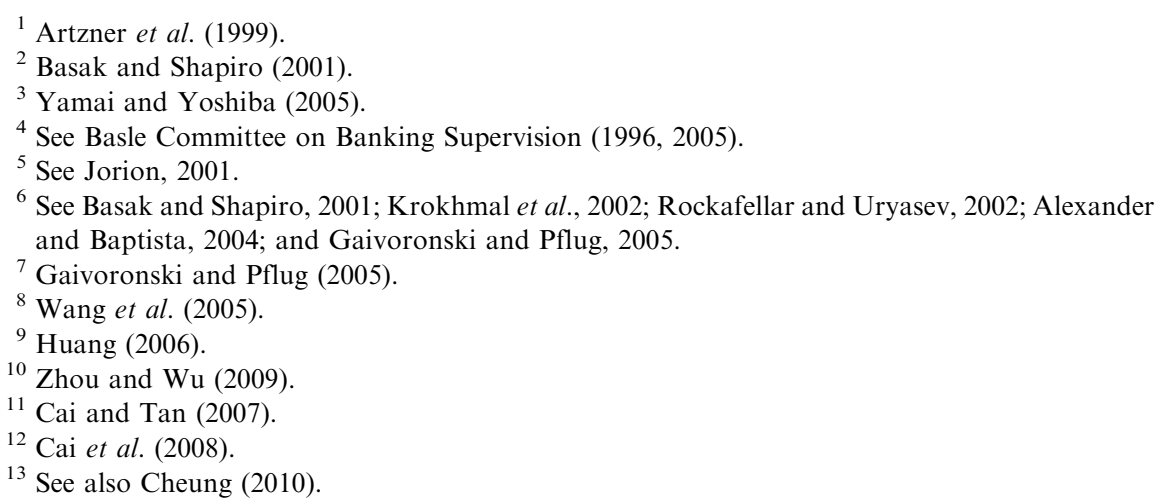


premium to the reinsurer upfront. Furthermore the greater the expected loss that is ceded to a reinsurer, the higher the reinsurance premium. The additional cost associated with the reinsurance has a direct impact on the overall profitability of the insurer. Hence, it is unsatisfactory to determine the optimal reinsurance treaty by just focusing on minimizing an insurer's risk exposure. A viable optimal reinsurance treaty should reflect both risk exposure and cost (or equivalently profitability); this is the classical risk and reward tradeoff.

The above argument implies that an insurer is faced with the conflicting objectives of risk transfer and profitability. Consequently, a more desirable optimal reinsurance model is the one that minimizes an insurer's risk exposure while at the same time taking into consideration its profitability. This is precisely the motivation of the proposed study in this paper. We formulate the optimal reinsurance model as a linear programme using $\mathrm{VaR}$ as the pertinent measure of the insurer's risk exposure. Furthermore, by confining to a class of increasing convex ceded loss function, we explicitly derive the optimal reinsurance policy. There are at least four reasons for imposing the ceded loss function being increasing and convex. The first reason is driven by the market practice. Commonly available reinsurance treaties such as quota-share reinsurance, stoploss reinsurance, and change-loss reinsurance (a combination of quota-share and stop-loss reinsurance) are increasing and convex. The second is motivated by the theoretical findings. Many well-known results in the literature have supported that the stop-loss type reinsurance treaty is optimal under various optimal reinsurance models. The third is due to the tractability and finally, without imposing the increasing and convex assumptions, the resulting optimal ceded loss function could be counter-intuitive and could even lead to moral hazard. We will further elaborate these reasons in the next section.

The remainder of the paper is organized as follows: the next section specifies the reinsurance model that is of interest to the paper. The section after that reformulates the proposed reinsurance model as an equivalent linear programming problem. The subsequent section presents the optimal solutions together with some remarks. We also provide two numerical examples to highlight and contrast the importance of incorporating the profitability constraint in our proposed reinsurance model. The last section concludes the paper. The technical details and the proofs of the main results are relegated to Appendix A and Appendix B.

\section{Optimal reinsurance model}

Let $X$ denote the (aggregate) loss initially assumed by an insurer. The random variable $X$ is assumed to be nonnegative with cumulative distribution function $F_{X}(x)=\operatorname{Pr}\{X \leqslant x\}$, survival function $S_{X}(x)=1-F_{X}(x)=\operatorname{Pr}\{X>x\}$, and mean 
$0<\mathrm{E}[X]<\infty$. To simplify our discussions, we assume that $X$ has a continuous strictly increasing distribution function on $(0, \infty)$ with a possible jump at 0 , which allows $X$ to be a random sum $\sum_{i=1}^{N} X_{i}$. This is an important special case in the actuarial loss model. ${ }^{11}$

Given the initial exposure to $X$, we assume that an insurer is interested in using reinsurance to manage its risk. Under the reinsurance arrangement, the insurer cedes part of its loss, denoted by $f(X)$, to a reinsurer while retaining the remaining loss $I_{f}(X)$. The function $f(x)$, satisfying the indemnity constraint $0 \leqslant f(X) \leqslant X$, is known as the ceded loss function and $I_{f}(x)=x-f(x)$ is the retained loss function. In exchange of ceding part of its loss to a reinsurer, the insurer incurs an upfront cost in the form of a reinsurance premium. By $\Pi_{f}(X)$ we denote as the resulting reinsurance premium for a given reinsurance policy $f$ written on the aggregate loss $X$, and by $T_{f}(X)$ we define as the sum of the retained loss and the reinsurance premium. In other words, we have the following relationship:

$$
T_{f}(X)=I_{f}(X)+\Pi_{f}(X)=X-f(X)+\Pi_{f}(X) .
$$

Reducing $T_{f}(X)$ by $\pi$, the aggregate insurance premium received by the insurer from the insureds (or policyholders) for insuring $X$, we obtain the net cost or the net risk of insuring risk $X$ in the presence of reinsurance. Using $N C_{f}(X)$ to denote the resulting net risk random variable, we have

$$
N C_{f}(X)=T_{f}(X)-\pi=X-f(X)+\Pi_{f}(X)-\pi .
$$

We now make three remarks with respect to $N C_{f}(X)$. First, it is more instructive to consider the random variable $N C_{f}(X)$ as opposed to the random variable $T_{f}(X)$ as in Cai and $\operatorname{Tan}^{11}$ and Cai et al. ${ }^{12}$ The random variable $T_{f}(X)$ can be interpreted as the total risk (or the total cost) of the insurer in the presence of reinsurance. $N C_{f}(X)$, on the other hand, takes into consideration both insurance premium inflow and reinsurance premium outflow. From a risk management point of view and the viability of the underlying business, it is therefore more prudent to focus on $N C_{f}(X)$. Second, (2) clearly demonstrates the intricate roles of the loss random variable $X$, the reinsurance policy $f$, the insurance premium $\pi$, and the reinsurance premium $\Pi_{f}(X)$ on $N C_{f}(X)$. In particular, the choice of the ceded loss function $f$ could have a tremendous impact on $N C_{f}(X)$ since for an initially insured loss $X$, the aggregate insurance premium $\pi$ is fixed but the reinsurance premium $\Pi_{f}$ is critically dependent on $f$. Third, the classical risk and reward is highlighted in (2). An insurer could reduce its risk exposure by transferring most of its expected risk to a reinsurer but at the expense of higher upfront reinsurance premium. On the other hand, if the insurer were to reduce its cost of reinsurance, this could be achieved by exposing to a higher risk exposure. The interplay between the ceded loss 
function $f$ and the reinsurance premium $\Pi_{f}$ and their overall effect on the net cost random variable $N C_{f}(X)$ are the key to the determination of the optimal reinsurance policy. An appropriate choice of ceded loss function could provide an effective way of reducing the risk exposure of an insurer.

Let us now briefly review $\mathrm{VaR}$ and some of its properties. Formally, the $\mathrm{VaR}$ of a random variable $X$ at a confidence level $1-\alpha, 0<\alpha<1$, is defined as

$$
\operatorname{VaR}_{\alpha}(X)=\inf \{x: \operatorname{Pr}\{X>x\} \leqslant \alpha\},
$$

where the parameter $\alpha$ is typically a small value such as 1 or 5 per cent. Note that if $X$ has a continuous strictly increasing distribution function on $(0, \infty)$, then we have $\operatorname{VaR}_{\alpha}(X)=S_{X}^{-1}(\alpha)$, where $S_{X}^{-1}$ is the inverse of the survival function $S_{X}$. In addition, the following two properties on the VaR will be useful in our subsequent discussions. If function is increasing and continuous, then

$$
\operatorname{VaR}_{\alpha}(f(X))=f\left(\operatorname{VaR}_{\alpha}(X)\right) .
$$

See Dhaene $e t a l .{ }^{14}$ for the proof of the above property. For any constant $c$, the VaR satisfies the translation invariance property; that is

$$
\operatorname{VaR}_{\alpha}(X-c)=\operatorname{VaR}_{\alpha}(X)-c .
$$

Exploiting $\mathrm{VaR}$ as a measure of risk exposure for an insurer, an optimal reinsurance design could be defined as the solution to the following optimization problem:

$$
\left\{\begin{array}{l}
\min _{f} \operatorname{VaR}_{\alpha}\left(N C_{f}(X)\right) \\
\text { subject to } E\left[-N C_{f}(X)\right] \geqslant P \\
\text { and } 0 \leqslant f(x) \leqslant x .
\end{array}\right.
$$

In the above formulation, the objective function $\operatorname{VaR}_{\alpha}\left(N C_{f}(X)\right)$ corresponds to the $\mathrm{VaR}$ of the net cost random variable $N C_{f}(X)$. Ideally the risk exposure of the insurer, as measured by $\operatorname{VaR}_{\alpha}\left(N C_{f}(X)\right)$, should be as low as possible for a chosen ceded loss function. The expectation $E\left[-N C_{f}(X)\right]$ can be interpreted as the expected profit and hence the inequality ensures that the expected profit of the insurer under the ceded loss function $f$ is at least $P$. The optimal reinsurance treaty that solves the above optimization problem (5) therefore minimizes the risk exposure of the insurer while guaranteeing a certain level of expected profit $P$. The second constraint $0 \leqslant f(x) \leqslant x$ ensures that the loss

\footnotetext{
${ }^{14}$ Dhaene et al. (2002).
} 
ceded to a reinsurer should be no larger than the loss incurred to the insurer and is typically known as the indemnity constraint.

The optimization problem (5) can be simplified further by first noting that the objective of minimizing $\operatorname{VaR}_{\alpha}\left(N C_{f}(X)\right)$ over function $f$ is equivalent to minimizing $\operatorname{VaR}_{\alpha}\left(T_{f}(X)\right)$. This is due to the property (4). Second, under the additional assumption that the reinsurance premium $\Pi_{f}(X)$ is determined by the expectation premium principle; that is,

$$
\Pi_{f}(X)=(1+\theta) E[f(X)],
$$

where $\theta>0$ is the safety loading factor, it follows from (2) and (6) that the profitability condition $E\left[-N C_{f}(X)\right] \geqslant P$ in (5) is equivalent to

$$
E[f(X)] \leqslant B \quad \text { with } \quad B=\frac{\pi-P-E[X]}{\theta} .
$$

Third, the minimization in (5) is assumed to be taken over the class of ceded loss functions $\mathcal{F}$ consisting of all increasing and convex functions $f(x)$ defined on $(0, \infty)$.

As alluded in the introduction, there are four reasons for imposing the increasing and convex conditions on the ceded loss function. First, these assumptions are consistent with practice in that the reinsurance treaties such as the quota-share reinsurance with $f(x)=a x$ and $I_{f}(x)=(1-a) x, 0<a \leqslant 1$, the stop-loss reinsurance with $f(x)=(x-d)_{+}=\max \{0, x-d\}$ and $I_{f}(x)=\min \{x, d\}$, $d \geqslant 0$, and the change-loss reinsurance (combination of quota-share and stoploss reinsurance) with $f(x)=a(x-d)_{+}$and $I_{f}(x)=(1-a) x+a \min \{x, d\}$ are common in the marketplace. All of these treaties are increasing and convex. Second, the reinsurance treaties mentioned above are by far the most widely analysed contracts in the literature. Just to name a few, it is well known that the stop-loss reinsurance is optimal in that it yields the lowest variance of retained loss among the class of ceded loss functions with the same expectations; see, for example, Bowers et al. ${ }^{15}$ Kass et al. ${ }^{16}$ and Gerber. ${ }^{17}$ By using the criterion of minimizing some specific convex risk measures, Gajek and Zagrodny ${ }^{18}$ demonstrated that the change-loss reinsurance is optimal. See also Gajek and Zagrodny ${ }^{19}$ and Kaluszka ${ }^{20}$ for other optimal reinsurance models.

\footnotetext{
${ }^{15}$ Bowers et al. (1997).

${ }^{16}$ Kass et al. (2001).

${ }^{17}$ Gerber (1979).

${ }^{18}$ Gajek and Zagrodny (2004).

${ }^{19}$ Gajek and Zagrodny (2000).

${ }^{20}$ Kaluszka (2001, 2005b).
} 
Third, this is driven by tractability. While it is of interest to consider the optimal solutions among all general reinsurance treaties, the resulting VaR optimization problem is notoriously non-tractable due to the lack of convexity. ${ }^{7}$ Some attempts have been conducted by Bernard and Tian $^{21}$ and Weng. ${ }^{22}$ However, the criterion adopted in Bernard and Tian $^{21}$ is only a VaRlike constraint, instead of the VaR risk measure in the strict definition. Weng ${ }^{22}$ explicitly adopts $\mathrm{VaR}$ in a reinsurance model but the optimal solutions are derived through a numerical method. Finally, not imposing the increasing condition on the ceded loss function could be counter-intuitive and could even lead to moral hazard. To elaborate, the results derived in both Bernard and Tian $^{21}$ and Weng ${ }^{22}$ indicate that the truncated stop-loss function could be optimal. The truncated stop-loss function has the peculiar property that for any loss less than a certain threshold level, the ceded loss function mimics a stop-loss in the sense that the amount ceded to the insurer is zero for loss less than the deductible and increases linearly for loss above the deductible until the threshold level. Beyond the threshold, the loss ceded to the reinsurer drops to zero. Such a phenomenon is counter-intuitive in that the insurer should be more concerned with extreme losses. Yet with the truncated stop-loss function, the insurer is only protected for moderate losses and no coverage for large extreme losses. In addition, there is a potential issue with the moral hazard induced by the truncated stop-loss function. See also Kaluszka, ${ }^{23}$ Kaluszka and Okolewski, ${ }^{24}$ and Bernard and Tian. ${ }^{21}$ For these reasons, we investigate the optimal ceded loss functions by confining to a class of increasing and convex functions $\mathcal{F}$.

In summary, the above arguments imply that the optimal reinsurance model (5) becomes

$$
\left\{\begin{array}{l}
\min _{f \in \mathcal{F}} \operatorname{VaR}_{\alpha}\left(T_{f}(X)\right) \\
\text { subject to } E[f(X)] \leqslant B \\
\text { and } 0 \leqslant f(x) \leqslant x \text { for all } x \geqslant 0 .
\end{array}\right.
$$

The indemnity constraint leads to $0 \leqslant E[f(X)] \leqslant E[X]$. This suggests that when $B \geqslant \mathrm{E}[X]$, the constrained optimization problem (8) reduces to an unconstrained problem since the profitability condition in (8) will have no effect. The optimal solution to this special case is addressed in Cai et al. ${ }^{12}$ The objective of this paper is to solve the optimal reinsurance model (8) and obtain explicitly the optimal reinsurance treaties, as discussed in the following sections.

\footnotetext{
${ }^{21}$ Bernard and Tian (2009).

${ }^{22}$ Weng (2009).

${ }^{23}$ Kaluszka (2005a).

${ }^{24}$ Kaluszka and Okolewski (2008).
} 
116

\section{Optimal reinsurance model reformulation}

As pointed out in Gaivoronski and Pflug, ${ }^{7}$ the optimization problem associated with $\mathrm{VaR}$, in general, is a non-trivial exercise. In order to obtain the solution of our proposed optimal reinsurance model, it is convenient to reformulate (8) as a linear programming with respect to $\sigma$-finite positive measures on the Borel measurable space $((0, \infty), \mathcal{B})$, where $\mathcal{B}:=\mathcal{B}(0, \infty)$ denotes the Borel sigma over the positive half real line $(0, \infty)$. To proceed, let us recall the following two important results, which we collect in the form of lemmas:

Lemma 1 An increasing convex function defined on $(0, \infty)$ can be represented as the following form:

$$
f(x)=f(0)+\int[x-t]_{+} d \mu, \text { for any fixed } x \geqslant 0,
$$

for some positive $\sigma$-additive measure $\mu$ on $\mathcal{B}$

Lemma 2 For any $f(x) \in \mathcal{F}, I_{f}(x)=x-f(x)$ is increasing and concave in $x$.

The proofs of these two lemmas can be found in Cardin and Pacelli ${ }^{25}$ and Cai et al. (Lemma A.1), ${ }^{12}$ respectively. We now make the following two remarks:

Remark 1 Note that for any ceded loss function $f \in \mathcal{F}, f(0)=0$ and hence by Lemma 1 the ceded loss function $f$ has the following representation:

$$
f(x)=\int(x-t)_{+} d \mu, \text { for any fixed } x \geqslant 0 .
$$

with a positive $\sigma$-finite measure $\mu$ on $\mathcal{B}$. Furthermore, by Fubini theory,

$$
E[f(X)]=\int E\left[(X-t)_{+}\right] d \mu .
$$

Remark 2 It follows from Lemma 2 that for any $f \in \mathcal{F}, I_{f}(x)$ is continuous and hence together with property (3), we have

$$
\begin{aligned}
\operatorname{VaR}_{\alpha}\left(T_{f}(X)\right) & =\operatorname{VaR}_{\alpha}(X-f(X)+(1+\theta) E[f(X)]) \\
& =\operatorname{VaR}_{\alpha}(X)-f\left(\operatorname{VaR}_{\alpha}(X)\right)+(1+\theta) E[f(X)] .
\end{aligned}
$$

Note that when $\alpha \geqslant S_{X}(0)$, then $\operatorname{VaR}_{\alpha}(X)=0$, the goal function $\operatorname{VaR}_{\alpha}\left(T_{f}(X)\right)$ in model (8) depends on $E[f(X)]$ and $\theta$ so that it is optimal for the insurer

\footnotetext{
${ }^{25}$ Cardin and Pacelli (2007).
} 
not to cede any risk. To avoid this trivial case, we assume $0<\alpha<S_{X}(0)$ hereafter.

Together with (10), (11) and setting $\phi(t)=\left(\operatorname{VaR}_{\alpha}(X)-t\right)_{+}$and $\psi(t)=E\left[(X-t)_{+}\right]$ for $t \geqslant 0$, (12) can be rewritten as

$$
\operatorname{VaR}_{\alpha}\left(T_{f}(X)\right)=\operatorname{VaR}_{\alpha}(X)-\int[\phi(t)-(1+\theta) \psi(t)] d \mu
$$

Consequently, our proposed optimal reinsurance model (8) is equivalent to the following linear programming with respect to the positive measure $\mu$ :

$$
\begin{cases}\min _{\mu \in \mathcal{M}^{+}} \operatorname{VaR}_{\alpha}(X)-\int[\phi(t)-(1+\theta) \psi(t)] d \mu \\ \text { subject to } \int \psi(t) d \mu(t) \leqslant B,\end{cases}
$$

where $\mathcal{M}^{+}$denotes the set of all $\sigma$-finite positive measure on the measurable space $((0, \infty), \mathcal{B})$ such that $0 \leqslant \int(x-t)_{+} d \mu(t) \leqslant x$ for all $x \geqslant 0$.

\section{Optimal solutions and examples}

The optimal solutions to the above linear programming (13) are formally derived in Appendix A. It should, however, be emphasized that solving (13) is non-trivial. We use an approximating approach, which is a routine approach regarding the optimization over a measured space. More specifically, this entails us first approximating (13) by a series of linear programming with optimization over a certain set of discrete measures with a particular structure (see (A.2) in Appendix A), and then reformulating the programming as some equivalent models (A.6), which are optimization problems over Euclidean space. By first obtaining the optimal solutions to (A.6) (and equivalently (A.2)), we then justify that these solutions are also the required solutions to (13) and (8) (or equivalently (5) with $f$ restricted in $\mathcal{F}$ ).

Table 1 summarizes the optimal solutions that are derived in Appendix A. The third column (with label $\mu^{*}$ ) and fourth column (with label $f^{*}(x)$ ) of the table give the solutions to models (13) and (8), respectively. To understand these results, we need to define some additional notations, which are

$$
\begin{gathered}
\rho=\frac{1}{1+\theta}, \delta_{\rho}=S_{X}^{-1}(\rho), \delta_{\alpha}=S_{X}^{-1}(\alpha), \\
\beta(d)=\frac{B}{\int_{d}^{\infty} S_{X}(x) d x},
\end{gathered}
$$


Table 1 Optimal ceded loss functions and minimal VaR for the seven possible cases that are analyzed in the Appendix A

\begin{tabular}{lllll}
\hline Case & Conditions & $\mu^{*}$ & $f^{*}(x)$ & VaR \\
\hline (i) & $\alpha \geqslant \rho$ & 0 & 0 & $\delta_{\alpha}^{*}$ \\
(ii) & $\alpha<\rho, \kappa(\delta \rho)>0$ & 0 & 0 & $\delta_{\alpha}$ \\
(iii) & $\alpha<\rho, \kappa(\delta \rho)=0$ & $c^{*} \mathcal{X}(\delta \rho, \cdot)$ where & $c^{*}(x-\delta \rho)_{+}$, for & $\delta_{\alpha}$ \\
& & $0 \leqslant c^{*} \leqslant \min \left\{\beta\left(\delta_{\rho}\right), 1\right\}$ & $0 \leqslant c^{*} \leqslant \min \left\{\beta\left(\delta_{\rho}\right), 1\right\}$ & \\
(iv) $\quad \alpha<\rho, \kappa\left(\delta_{\rho}\right)<0, \beta\left(\delta_{\rho}\right)>1$ & $\mathcal{X}\left(\delta_{\rho}, \cdot\right)$ & $\left(x-\delta_{\rho}\right)_{+}$ & $\delta_{\alpha}+\kappa\left(\delta_{\rho}\right)$ \\
(v) $\quad \alpha<\rho, \kappa\left(\delta_{\rho}\right)<0$, & $\beta\left(\delta_{\rho}\right) \mathcal{X}\left(\delta_{\rho}, \cdot\right)$ & $\beta\left(\delta_{\rho}\right)\left(x-\delta_{\rho}\right)_{+}$ & $\delta_{\alpha}+\beta\left(\delta_{\rho}\right) \kappa\left(\delta_{\rho}\right)$ \\
& $\beta\left(\delta_{\rho}\right) \leqslant 1, \lambda\left(\delta_{\rho}\right) \geqslant 0$ & $\beta\left(d_{o}\right) \mathcal{X}\left(d_{o}, \cdot\right)$ & $\beta\left(d_{o}\right)\left(x-d_{o}\right)_{+}$ & $\delta \alpha-B((1) /$ \\
(vi) $\quad \alpha<\rho, \kappa\left(\delta_{\rho}\right)<0$, & $\left(x-d_{B}\right)_{+}$ & $\left.\left(S_{X}(d o)\right)-(1) /(\rho)\right)$ \\
& $\beta\left(\delta_{\rho}\right) \leqslant 1, \lambda\left(\delta_{\rho}\right)<0, \beta\left(d_{o}\right) \leqslant 1$ & $\mathcal{X}\left(d_{B}, \cdot\right)$ & $\delta_{\alpha}+u\left(d_{B}\right)$
\end{tabular}

Column 2 states the conditions for each case. For each case, column 3 gives the optimal measure to problems (13) and (A.2), while column 4 presents the corresponding optimal ceded loss function to the model (8). Column 5 tabulates the minimal value of $\operatorname{VaR}_{\alpha}\left(T_{f}(X)\right)$.

$$
\begin{aligned}
& \kappa(d)=d+(1+\theta) \int_{d}^{\infty} S_{X}(x) d x-\delta_{\alpha}, \\
& \lambda(d)=\int_{d}^{\infty} S_{X}(x) d x+S_{X}(d)\left(d-\delta_{\alpha}\right),
\end{aligned}
$$

$\mathcal{X}(d, \cdot)$ is the Dirac measure concentrated at the point $d, d_{o}$ is the unique solution to $\lambda(d)=0$ (see Lemma B.1(b) in Appendix B) and $d_{B}$ satisfies the following equation:

$$
B=\int_{d_{B}}^{\infty} S_{X}(x) d x .
$$

Furthermore, the $\mathrm{VaR}_{\alpha}^{*}$ in column five is the corresponding minimal $\operatorname{VaR}_{\alpha}\left(T_{f}(X)\right)$ for $f$ over the class of increasing and convex functions such that $0 \leqslant f(x) \leqslant x, \forall x \geqslant 0$. Note that the optimal ceded loss function $f^{*}(x)$ is related to $\mu^{*}$ via (10).

As pointed out earlier that Cai et al. ${ }^{12}$ proposed an unconstrained optimal reinsurance model that determines the optimal ceded loss function by minimizing certain risk measures of the insurer's total risk exposure. The approach described in this paper, on the other hand, is a generalization of their model in the sense that we impose an additional profitability constraint. The model proposed in this paper is intuitively more appealing since it takes into account both risk and 
reward, consistent with practice. We now make the following remarks to compare and contrast the results obtained in this paper to that in Cai et al. ${ }^{12}$

Remark 3 Except for the first two cases, the optimal ceded loss functions presented in Table 1 are all in the forms of stop-loss types. In fact, under certain conditions they reduce to the quota-share treaties. For example, if $\rho>S(0)$, then $\delta_{\rho} \equiv S_{X}^{-1}(\rho)=0$, which implies that the optimal ceded loss functions in Cases (iii), (iv), and (v) collapse to the quota-share type.

Remark 4 Recall that when $B>E[X]$, the expected profit constraint in our proposed constrained optimal reinsurance model will have no impact on the solution. Consequently, this special case reduces to the results of Cai et al. ${ }^{12}$ We can also examine the range of insurers' expected profit $P$ corresponding to this special case. Note that when $B>E[X]$, the inequality $B>\int_{\delta_{\rho}}^{\infty} S_{X}(x) d x$ (or equivalently $\beta\left(\delta_{\rho}\right)>1$ ) holds trivially. Furthermore, the condition $B>\int_{\delta_{\rho}}^{\infty} S_{X}(x) d x$ implies

$$
P<\pi-E[X]-\theta \int_{\delta_{\rho}}^{\infty} S_{X}(x) d x .
$$

Hence if the expected profit $P$ of the insurer is less than the quantity on the right hand side of the above inequality, the profitability constraint becomes redundant. In fact, in this situation, Case (iv) in Table 1 recovers Theorem 3.1, parts (a) and (c) of Cai et al. ${ }^{12}$ while Case (iii) in Table 1 is equivalent to Theorem 3.1, parts (b) and (d) of Cai et al. ${ }^{12}$

Remark 5 To understand the impact of imposing the profitability constraint on the optimal reinsurance model, let us compare Case (iv) to Case (v) and assume $\lambda\left(\delta_{\rho}\right) \geqslant 0$. When an insurer becomes more aggressive so that it requires an expected profit greater than the quantity on the right-hand-side of the inequality (19) (i.e. $\beta\left(\delta_{\rho}\right)<1$ ), the optimal ceded loss function is $f^{*}(x)=$ $\beta\left(\delta_{\rho}\right)\left(x-\delta_{\rho}\right)_{+}$with $\mathrm{VaR}_{\alpha}^{*}=\delta_{\alpha}+\beta\left(\delta_{\rho}\right) \cdot \kappa\left(\delta_{\rho}\right)$. By contrasting these results to the unconstrained model as in Table 1, Case (iv) implies that under the profitability constraint, the optimal reinsurance design is to retain greater loss while expose to a higher minimum attainable $\mathrm{VaR}_{\alpha}^{*}$. This is consistent with the classical risk and reward tradeoff.

To conclude this section, we provide two examples to illustrate our results.

Example 1 Assume $X$ is exponentially distributed with mean $E[X]=1,000$. Then $S_{X}(x)=e^{-0.001 x}, x \geqslant 0$, and $S_{X}(0)=1$. Assume further that the loading factors for the reinsurer and insurer are 20 and 15 per cent, respectively. This implies $\theta=0.2$ and $\delta_{\rho}=S_{X}^{-1}(1 /(1+\theta))=182.32$. Under the additional assumption of the expectation premium principle, we have $\pi=1.15 E[X]=1150$ and 
Table $2 \mathrm{VaR}_{\alpha}{ }^{*}$ and optimal ceded loss functions: Exponential risk

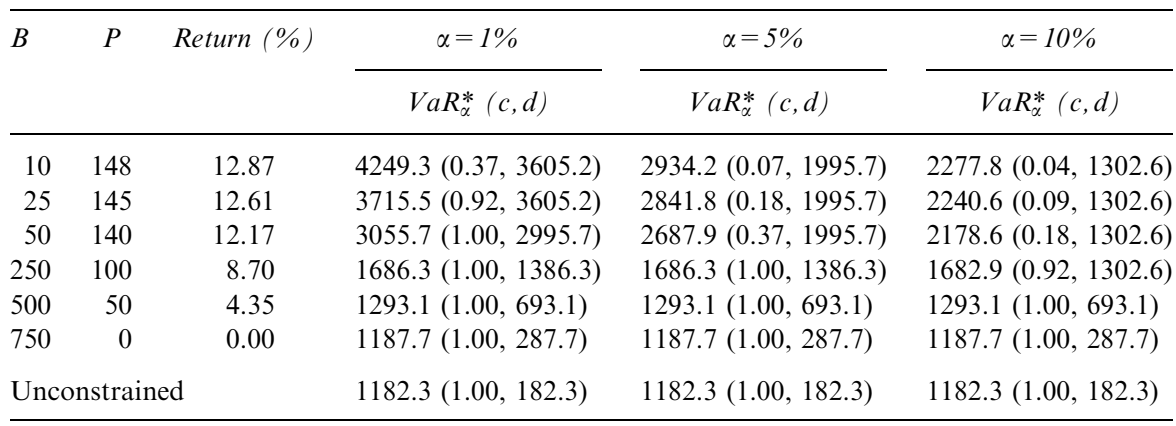

$\Pi_{f}(X)=1.2 E[f(X)]$ for a given ceded loss function. In practice it is to be expected that the loading factor for the reinsurer is higher than the insurer's. Consequently, the achievable expected profits $P$ are in the range $[0,150]$ so that $B \in[0,750]$. Table 2 reports the $\mathrm{VaR}_{\alpha}^{*}$ and the corresponding optimal ceded loss function (as specified by $c$ and $d$ ) for different combinations of $P \in\{148,145,140,100,50,0\}$ and $\alpha \in\{1$ per cent, 5 per cent, 10 per cent $\}$. For these parameter values, conditions $\alpha<\rho, \kappa\left(\delta_{\rho}\right)<0$ and $\beta\left(\delta_{\rho}\right) \leqslant 1$ are satisfied and hence Proposition A.4 is used to determine the optimal solutions. For example, at $\alpha=10$ per cent the root of $\lambda(d)$ occurs at $d_{o}=1302.6$ with $\beta\left(d_{o}\right)$ depends on the level of $B$ (see Eq. (15). By guaranteeing an expected profit of US\$145 (or equivalently $B=25$ ), the optimal ceded loss function in $\mathcal{F}$ becomes a combination of quota-share and stop-loss reinsurance given by $f(x)=c(x-d)_{+}$where $c=\beta\left(d_{o}\right)=0.09, d=d_{o}=1302.6$ and with minimum attainable VaR US\$2240.6.

Note that when we increase the confidence level $1-\alpha$, the minimum VaR, and the optimal values of $c$ and $d$ become larger as long as $\beta\left(d_{o}\right) \leqslant 1$. This implies that higher level of confidence can be achieved at the expense of higher exposure to risk (i.e. higher VaR). Furthermore, the optimal reinsurance contract and the minimum attainable $\mathrm{VaR}$ are invariant to $\alpha$ as long as $\beta\left(d_{o}\right)>1$.

The impact of the expected profit $P$ (or equivalently $B$ ) on optimal reinsurance is also clearly demonstrated. First, if we were to decrease the minimum level of the expected profits, the optimal retention $d$ does not change as long as $\beta\left(d_{o}\right) \leqslant 1$. The optimal $c$, however, increases accordingly as asserted in part (i), Proposition A.4(b). This is also consistent with our numerical results. Second, when the condition $\beta\left(d_{o}\right)>1$ is satisfied as we further decrease $P$, the optimal reinsurance design becomes a pure stop-loss contract with the optimal retention $d$ that also declines with $P$ (see Eq. (18)). Third, the minimum attainable $\mathrm{VaR}$ is an increasing function in $P$. This is the classical risk and reward tradeoff in the sense that higher expected profit can be achieved at the 
Table 3 VaR ${ }_{\alpha}^{*}$ and optimal ceded loss functions: Pareto risk

\begin{tabular}{|c|c|c|c|c|c|}
\hline \multirow[t]{2}{*}{$B$} & \multirow[t]{2}{*}{$P$} & \multirow[t]{2}{*}{ Return (\%) } & $\alpha=1 \%$ & $\alpha=5 \%$ & $\alpha=10 \%$ \\
\hline & & & $\operatorname{VaR}_{\alpha}^{*}(c, d)$ & $\operatorname{VaR}_{\alpha}^{*}(c, d)$ & $\operatorname{VaR}_{\alpha}^{*}(c, d)$ \\
\hline 10 & 148 & 12.87 & $6998.9(0.10,4188.8)$ & $3381.6(0.03,1619.2)$ & $2291.2(0.02,872.6)$ \\
\hline 25 & 145 & 12.61 & $6572.4(0.24,4188.8)$ & $3310.7(0.08,1619.2)$ & $2264.8(0.05,872.6)$ \\
\hline 50 & 140 & 12.17 & $5861.7(0.48,4188.8)$ & $3192.5(0.16,1619.2)$ & $2220.7(0.10,872.6)$ \\
\hline 250 & 100 & 8.70 & $2300.0(1.00,2000.0)$ & $2247.4(0.82,1619.2)$ & $1868.1(0.52,872.6)$ \\
\hline 500 & 50 & 4.35 & $1428.4(1.00,828.4)$ & $1428.4(1.00,828.4)$ & $1428.4(1.00,828.4)$ \\
\hline 750 & 0 & 0.00 & $1209.4(1.00,309.4)$ & $1209.4(1.00,309.4)$ & $1209.4(1.00,309.4)$ \\
\hline \multicolumn{3}{|c|}{ Unconstrained } & $1188.0(1.00,125.3)$ & $1188.0(1.00,125.3)$ & $1188.0(1.00,125.3)$ \\
\hline
\end{tabular}

expense of higher minimum risk exposure (as measured by the VaR); see Remark 5. Fourth, if we were to permit $B$ to increase beyond 833.33 (and $P$ is negative), then $\beta\left(\delta_{\rho}\right)>1$ since $\int \delta_{\rho}^{\infty} S_{X}(x) d x=833.33$ and part (b) of Proposition A.3 can be used to determine the optimal ceded loss function. In this case, the upper constraint $B$ has no impact on the optimization problem and it reduces to the unconstrained problem, as studied in Cai et al. ${ }^{12}$ In our example, VaR ${ }_{\alpha}^{*}$ is US\$1182.3 with optimal retention $\delta_{\rho}=182.3$. See also Remark 4. The unconstrained optimal reinsurance design also serves as a benchmark to our proposed constrained optimization problem. For instance at $\alpha=1$ per cent, if the insurer were to seek an expected profit of US\$145, the insurer would need to sustain more than three times the risk exposure relative to the unconstrained case (compare US\$3715.5 to 1182.3 ).

Example 2 In this example, we assume $X$ has a Pareto distribution with $S_{X}(x)=((2,000) /(x+2,000))^{3}, x \geqslant 0$ so that its $E[X]=1,000$ is the same as the previous example. We also assume $\theta=0.2$ and $\pi=1150$. Table 3 produces the optimal reinsurance designs over the same set of parameter values as in the last example. Note that for a given $P$ and $\alpha$, the minimum attainable VaR is larger for the Pareto risk. This is to be expected since Pareto distribution is considered to be riskier than the corresponding exponential distribution in the sense that it has a heavier tail. Other than this, the discussions and the conclusions that we made in the last example are equally applicable to this example.

\section{Conclusion}

In this paper, we extended and improved the optimal reinsurance model of Cai et al. ${ }^{12}$ by formulating the model as a constrained optimization problem. By minimizing the $\mathrm{VaR}$ of the total risk of an insurer while subjecting to a 
profitability constraint, explicit solutions for the optimal reinsurance were derived. We formally established that the optimal reinsurance could be in the form of a pure stop-loss reinsurance, a quota-share reinsurance, a combination of them, or not to cede at all, depending on the risk measure's level of confidence, the safety loading for the reinsurance premium, and the expected profit guaranteed for the insurer. The numerical examples highlighted the importance of incorporating the profitability condition.

The key assumptions in our proposed optimal reinsurance model are (i) reinsurance premium is determined by the expectation premium principle, (ii) ceded loss function is confined to a class of increasing and convex functions, and (iii) risk exposure of the insurer is captured by its VaR measure. It will be of interest to investigate the impact on the optimal reinsurance design if any of these assumptions are modified.

The expectation premium principle is a linear function. Incorporating other premium principles in a reinsurance model usually incurs additional mathematical complexity. In particular, when the objective is to minimize the VaR risk measure, a nonlinear reinsurance premium principle usually results in a notoriously nontractable model. At this point, it might be interesting to mention the thesis by Weng, ${ }^{22}$ who developed a numerical method in analysing the optimal solution to the VaR minimization model. ${ }^{26}$ His proposed numerical solution, however, is only applicable to the expectation reinsurance premium principle. Consequently, exploring the optimal solution to the VaR-based reinsurance models under general premium principles is an ongoing and challenging problem.

Besides VaR, there are other risk measures such as the CTE that can be incorporated in the proposed reinsurance model. See for example Gajek and Zagrodny ${ }^{18}$ and Balbás et al. ${ }^{27}$ However, it should be emphasized that the CTE minimization reinsurance model is usually much more tractable due to the convexity of CTE. In fact under the expectation reinsurance premium principle, Tan et al. ${ }^{28}$ explicitly derived the optimal solutions over the class of all general reinsurance treaties. Their results again confirm that a stop-loss reinsurance treaty is optimal.

\section{Acknowledgements}

This research is supported by the MOE Project of Key Research Institute of Humanities and Social Sciences at Universities (11JJD790004) and NSERC. Weng acknowledges research support from the Natural Sciences and Engineering Research Council of Canada.

\footnotetext{
${ }^{26}$ See Weng (2009, Section 5.7).

${ }^{27}$ Balbás et al. (2009).

${ }^{28}$ Tan et al. (2011).
} 


\section{References}

Alexander, G.J. and Baptista, A.M. (2004) 'A comparison of VaR and CVaR constraints on portfolio selection with the mean-variance model', Management Science 50(9): 1261-1273.

Artzner, P., Delbaen, F., Eber, J. and Heath, D. (1999) 'Coherent measures of risk', Mathematical Finance 9: 203-228.

Balbás, A., Balbás, B. and Heras, A. (2009) 'Optimal reinsurance with general risk measures', Insurance: Mathematics and Economics 44(3): 374-384.

Basak, S. and Shapiro, A. (2001) 'Value-at-risk based risk management: Optimal policies and asset prices', Review of Financial Studies 14(2): 371-405.

Basle Committee on Banking Supervision (1996) Amendment to the Capital Accord to Incorporate Market Risks, Basle, Switcherland: Basle Committee on Banking Supervision.

Basle Committee on Banking Supervision (2005) 'Basel II: International convergence of capital measurement and capital standards: A revised framework', www.bis.org, Basle, Switcherland.

Bernard, C. and Tian, W. (2009) 'Optimal reinsurance arrangements under tail risk measures', The Journal of Risk and Insurance 76(3): 709-725.

Bowers, N.J., Gerber, H.U., Hickman, J.C., Jones, D.A. and Nesbitt, C.J. (1997) Actuarial Mathematics, 2nd edn, Schaumburg: The Society of Actuaries.

Cai, J. and Tan, K.S. (2007) 'Optimal retention for a stop-loss reinsurance under the VaR and CTE risk measure', Astin Bulletin 37(1): 93-112.

Cai, J., Tan, K.S., Weng, C. and Zhang, Y. (2008) 'Optimal reinsurance under VaR and CTE risk measures', Insurance: Mathematics and Economics 43(1): 185-196.

Cardin, M. and Pacelli, G. (2007) 'Characterization of convex premium principles', in C. Perna and M. Sibillo (eds.) Mathematical and Statistical Methods in Insurance and Finance, New York: Springer, pp. 53-60.

Cheung, K.C. (2010) 'Optimal reinsurance revisited - A geometric approach', Astin Bulletin 40(1): 221-239.

Dhaene, J., Denuit, M., Goovaerts, M.J., Kaas, R. and Vyncke, D. (2002) 'The concept of comonotonicity in actuarial science and finance: Theory', Insurance: Mathematics and Economics 31(1): 3-33.

Gaivoronski, A.A. and Pflug, G. (Winter 2004-2005) 'Value-at-Risk in portfolio optimizaiton: Properties and computational approach', Journal of Risk 7(2): 1-31.

Gajek, L. and Zagrodny, D. (2000) 'Insurer's optimal reinsurance strategies', Insurance: Mathematics and Economics 27: 105-122.

Gajek, L. and Zagrodny, D. (2004) 'Optimal reinsurance under general risk measures', Insurance: Mathematics and Economics 34: 227-240.

Gerber, H.U. (1979) An Introduction to Mathematical Risk Theory, Philadelphia: S.S. Huebner Foundation for Insurance Education, Wharton School, University of Pennsylvania.

Huang, H.H. (2006) 'Optimal insurance contract under a value-at-risk constraint', The Geneva Risk and Insurance Review 31: 91-110.

Jorion, P. (2001) Value at Risk: The New Benchmark for Managing Financial Risk, New York: McGraw-Hill.

Kass, R., Goovaerts, M., Dhaene, J. and Denuit, M. (2001) Modern Actuarial Risk Theory, Boston: Kluwer Academic Publishers.

Kaluszka, M. (2001) 'Optimal reinsurance under mean-variance premium principles', Insurance: Mathematics and Economics 28: 61-67.

Kaluszka, M. (2005a) 'Truncated stop loss as optimal reinsurance agreement in one-period models', ASTIN Bulletin 35(2): 337-349.

Kaluszka, M. (2005b) 'Optimal reinsurance under convex principles of premium calculation', Insurance: Mathematics and Economics 36: 375-398. 
Kaluszka, M. and Okolewski, A. (2008) 'An extension of Arrow's result on optimal reinsurance contract', The Journal of Risk and Insurance 75(2): 275-288.

Krokhmal, P., Palmquist, J. and Uryasev, S. (2002) 'Portfolio optimization with conditional value-at-risk objective and constraints', The Journal of Risk 4(2): 11-27.

Müller, A. and Stoyan, D. (2002) Comparison Methods for Stochastic Models and Risks, New York: Wiley.

Rockafellar, R.T. and Uryasev, S. (2002) 'Conditional value-at-risk for general loss distributions', Journal of Banking and Finance 26: 1443-1471.

Tan, K.S., Weng, C. and Zhang, Y. (2011) 'Optimality of general reinsurance contracts under CTE risk measure,' Insurance: Mathematics and Economics 49(2): 175-187.

Wang, C.P., Shyu, D. and Huang, H.H. (2005) 'Optimal insurance design under a value-at-risk framework', The Geneva Risk and Insurance Review 30: 161-179.

Weng, C. (2009) Optimal reinsurance designs: From an insurer's perspective, $\mathrm{PhD}$. Thesis, University of Waterloo.

Yamai, Y. and Yoshiba, Y. (2005) 'Value-at-risk versus expected shortfall: A practical perspective', Journal of Banking and Finance 29: 997-1015.

Zhou, C. and Wu, C. 2009 'Optimal insurance under the insurer's VaR constraint', The Geneva Risk and Insurance Review 34: 140-154.

\section{About the Authors}

Ken Seng Tan is a University Research Chair Professor in the Department of Statistics and Actuarial Science, University of Waterloo, Canada and Cheung Kong Scholar in the China Institute for Actuarial Science, Central University of Finance and Economics, Beijing, China.

Chengguo Weng is an Assistant Professor in the Department of Statistics and Actuarial Science, University of Waterloo, Canada.

\section{Appendix A}

\section{Derivation of the optimal reinsurance solutions}

This appendix is concerned with deriving the analytic solution to the linear programming (13). Before we delve into the mathematical details, we first briefly describe the approach that is used to obtain the desired result. Our strategy requires using an approximation approach, which is a standard approach with regard to optimization over a measure space. We first construct a linear programming of the form (A.2), which is an optimization problem over a set of discrete measures with special structure. This optimization is then reformulated as an equivalent optimization problem (A.6), which results in an optimization problem over the Euclidean space. Because (A.6) is now an optimization problem over the Euclidean space, the optimal solutions can be sought indirectly by subpartitioning the feasible set, as argued in (A.7). Propositions A.1-A.4 are devoted to analysing the optimal solutions for these partitions and hence the optimal 
solutions to (A.6), or equivalently (A.2). Finally, we conclude the appendix by demonstrating that the optimal solutions to (A.2) are also the optimal solutions to the proposed optimal reinsurance model (13).

For integer $n \geqslant 1$, let $\mathcal{M}_{n}^{+}$denote the set of all measures on $((0, \infty), \mathcal{B})$ with the following structure:

$$
\mu_{n}(\cdot)=\sum_{j=1}^{n} c_{n, j} \mathcal{X}\left(d_{n, j}, \cdot\right), n \geqslant 1,
$$

where the coefficients $c_{n, j} \geqslant 0$ and $d_{n, j} \geqslant 0, j=1, \ldots, n, \sum_{j=1}^{n} c_{n, j} \leqslant 1$, and $\mathcal{X}\left(d_{n, j}, \cdot\right)$ is the Dirac measure concentrated on the point $d_{n, j}$. Without any loss of generality, we assume $0 \leqslant d_{n, 1} \leqslant d_{n, 2} \leqslant \cdots \leqslant d_{n, n}$ for all $n=1,2, \ldots$ Note that $\mathcal{M}_{n}^{+} \subset \mathcal{M}^{+}$. We now consider the following problems:

$$
\begin{cases}\min _{\mu_{n} \in \mathcal{M}_{n}^{+}} & \operatorname{VaR}_{\alpha}(X)-\int[\phi(t)-(1+\theta) \psi(t)] d \mu_{n} \\ \text { subject to } & \int \psi(t) d \mu_{n} \leqslant B .\end{cases}
$$

By defining coefficient vectors $\boldsymbol{c}:=\left(c_{n, 1}, c_{n, 2}, \ldots, c_{n, n}\right)$ and $\boldsymbol{d}:=\left(d_{n, 1}, d_{n, 2}, \ldots\right.$, $d_{n, n}$ ), and using (A.1), the goal function in (A.2) can be expressed as

$$
\begin{aligned}
\operatorname{VaR}_{\alpha}(\boldsymbol{c}, \boldsymbol{d}): & =\operatorname{VaR}_{\alpha}(X)-\int[\phi(t)-(1+\theta) \psi(t)] d \mu_{n} \\
& =\operatorname{VaR}_{\alpha}(X)-\sum_{j=1}^{n} c_{n, j}\left[\phi\left(d_{n, j}\right)-(1+\theta) \psi\left(d_{n, j}\right)\right] \\
& =\left\{\begin{array}{ll}
\delta_{\alpha}+\Pi_{\mu_{n}}(X), & \delta_{\alpha} \leqslant d_{n, 1}, \\
A_{n, i} \delta_{\alpha}+B_{n, i}+\Pi_{\mu_{n}}(X), & d_{n, i} \leqslant \delta_{\alpha} \leqslant d_{n, i+1}, \\
A_{n, n} \delta_{\alpha}+B_{n, n}+\Pi_{\mu_{n}}(X), & i=1, \ldots, n-1,
\end{array},\right.
\end{aligned}
$$

where

$$
\begin{aligned}
& A_{n, i}=1-\sum_{j=1}^{i} c_{n, j}, \\
& B_{n, i}=\sum_{j=1}^{i} c_{n, j} d_{n, j}, \quad i=1, \ldots, n,
\end{aligned}
$$

and

$$
\Pi_{\mu_{n}}(X)=(1+\theta)\left\{\sum_{j=1}^{n} c_{n, j} \int_{d_{n, j}}^{\infty} S_{X}(x) d x\right\} .
$$


126

Similarly, the constraint in the optimization problem (A.2) becomes

$$
\sum_{j=1}^{n} c_{n, j} \int_{d_{n, j}}^{\infty} S_{X}(x) d x \leqslant B .
$$

Note that the objective function and the constraint depend explicitly on variables $\boldsymbol{c}$ and $\boldsymbol{d}$. This explains the notation $\operatorname{VaR}_{\alpha}(\boldsymbol{c}, \boldsymbol{d})$ with the arguments $\boldsymbol{c}$ and $\boldsymbol{d}$.

Let us now introduce the following sets:

$$
\begin{aligned}
& C_{n}=\left\{\left(c_{n, 1}, \ldots, c_{n, n}\right) \in \mathbb{R}^{n}: c_{n, j} \geqslant 0, j=1,2, \ldots, n, \text { and } \sum_{j=1}^{n} c_{n, j} \leqslant 1\right\}, \\
& D_{n}=\left\{\left(d_{n, 1}, \ldots, d_{n, n}\right) \in \mathbb{R}^{n}: 0 \leqslant d_{n, 1} \leqslant \cdots \leqslant d_{n, n}\right\}, \\
& S_{n}=\left\{(\boldsymbol{c}, \boldsymbol{d}):(\boldsymbol{c}) \in C_{n},(\boldsymbol{d}) \in D_{n}, \sum_{j=1}^{n} c_{n, j} \int_{d_{n, j}}^{\infty} S_{X}(x) d x \leqslant B\right\} .
\end{aligned}
$$

Then the coefficient vectors $(\boldsymbol{c}, \boldsymbol{d})$ defined in (A.1) must satisfy $\boldsymbol{c} \in C_{n}$ and $\boldsymbol{d} \in D_{n}$. Furthermore, the set $S_{n}$ incorporates both feasible values of $\boldsymbol{c}$ and $\boldsymbol{d}$ as well as the constrained condition in problem (A.2). Consequently, the optimization problem (A.2) can be expressed more compactly as

$$
\min _{(\boldsymbol{c}, \boldsymbol{d}) \in S_{n}} \operatorname{VaR}_{\alpha}(\boldsymbol{c}, \boldsymbol{d}) \text {. }
$$

We now make several remarks with respect to the above optimization problem:

- The above optimization is a constrained optimization problem as the constraints on $\boldsymbol{c}$ and $\boldsymbol{d}$ are embedded in the definition of $S_{n}$.

- The above optimization is a problem of $2 n$ dimensions since for a given $n$, it involves determining the optimal vectors $(\boldsymbol{c}, \boldsymbol{d})$, each of $n$ dimensions.

- When $\boldsymbol{c}=\mathbf{0}=(0, \ldots, 0)$, where $\mathbf{0}$ is a zero vector of $n$ dimensions, the objective function and the constraint in (A.6) are constant and do not depend on $\boldsymbol{d}$. Furthermore, if $\boldsymbol{c}=\mathbf{0}$ is the optimal solution, then we have a situation where it is never optimal for the insurer to reinsure its risk.

- When $d_{n, j}=d$ for $j=1,2, \ldots$, and $d$ is a constant, both the objective function and the constraint depend on $d$ as well as the sum $c:=\sum_{j=1}^{n} c_{n, j}$ (i.e. independent of the allocations $\left.\left(c_{n 1}, c_{n 2}, \ldots, c_{n n}\right)\right)$. More importantly, in this special case the optimization problem (A.6) reduces to a two-dimensional problem of determining $c$ and $d$. In the sequel, we will simply denote $(c, d) \in S$ for this situation for a set structured in the same way as $S_{n}$. 
As pointed out earlier, the VaR-based optimization model, in general, is a non-trivial problem. ${ }^{7}$ It is, therefore, difficult to obtain the global minimizer of the constrained optimization problem (A.6) directly. On the other hand, the fact that (A.6) is now formulated as an optimization problem over the Euclidean space suggests that we can derive the optimal solution via the following approach. To explain this approach, let us first note that for $n=1,2, \ldots$, the sets $D_{n}$ and $S_{n}$ can be partitioned, respectively, as

$$
\begin{gathered}
D_{n}^{0}=\left\{\left(d_{n, 1}, \ldots, d_{n, n}\right) \in \mathbb{R}^{n}: \delta_{\alpha} \leqslant d_{n, 1} \leqslant \cdots \leqslant d_{n, n}\right\}, \\
D_{n}^{i}=\left\{\left(d_{n, 1}, \ldots, d_{n, n}\right) \in \mathbb{R}^{n}: 0 \leqslant d_{n, 1} \leqslant \cdots \leqslant d_{n, i} \leqslant \delta_{\alpha} \leqslant d_{n, i+1} \leqslant \cdots \leqslant d_{n, n}\right\}, \\
i=1, \ldots, n-1, \\
D_{n}^{n}=\left\{\left(d_{n, 1}, \ldots, d_{n, n}\right) \in \mathbb{R}^{n}: 0 \leqslant d_{n, 1} \leqslant \cdots \leqslant d_{n, n} \leqslant \delta_{\alpha}\right\},
\end{gathered}
$$

and

$$
\begin{aligned}
S_{n}^{i}= & \left\{(c, d): c \in C_{n}, d \in D_{n}^{i}, \sum_{j=1}^{n} c_{n, j} \int_{d_{n, j}}^{\infty} S_{X}(x) d x \leqslant B\right\}, \\
& i=0,1,2, \ldots, n .
\end{aligned}
$$

In other words, we have $D_{n}=\bigcup_{i=0}^{n} D_{n}^{i}$ and $S_{n}=\bigcup_{i=0}^{n} S_{n}^{i}$. The partition of $S_{n}$ into $S_{n}^{i}, i=0,1, \ldots, n$ enables us to analyse, case by case, the solution to (A.6) with the feasible set replaced by $S_{n}^{i}, i=0,1, \ldots, n$. The global solution to (A.6) over the feasible set $S_{n}$ is then given by the partition that yields the lowest VaR of the insurer's total risk among all partitions $S_{n}^{i}, i=0, \ldots, n$. More specifically, let $\operatorname{VaR}_{\alpha}^{*}(S)$ denote the minimum value of $\operatorname{VaR}_{\alpha}(\boldsymbol{c}, \boldsymbol{d})$ for $(\boldsymbol{c}, \boldsymbol{d})$ over the feasible set $S$ and let $\left(c^{*}, d^{*}\right) \in S$ be the corresponding optimal vectors for which the minimum is attained. Adopting this notation, $\operatorname{VaR}_{\alpha}^{*}\left(S_{n}\right)$ with optimal vectors $\left(c^{*}, d^{*}\right) \in S_{n}$ is the optimal solution to (A.6). The argument provided above implies that the minimum value $\operatorname{VaR}_{\alpha}^{*}\left(S_{n}\right)$ can be obtained indirectly via

$$
\operatorname{VaR}_{\alpha}^{*}\left(S_{n}\right)=\min \left\{\operatorname{VaR}_{\alpha}^{*}\left(S_{n}^{0}\right), \operatorname{VaR}_{\alpha}^{*}\left(S_{n}^{1}\right), \ldots, \operatorname{VaR}_{\alpha}^{*}\left(S_{n}^{n}\right)\right\},
$$

with the optimal vectors $\left(c^{*}, \boldsymbol{d}^{*}\right)$ correspond to the partition that yields the lowest VaR.

The remainder of this appendix is devoted to analysing the minimum value of $\operatorname{VaR}_{\alpha}(\boldsymbol{c}, \boldsymbol{d})$ for $(\boldsymbol{c}, \boldsymbol{d})$ over feasible sets $S_{n}^{i}, i=0,1,2, \ldots, n$. It turns out 
that the optimality associated with the first $n$ cases (i.e. for feasible sets $S_{n}^{i}$, $i=0, \ldots, n-1)$ is relatively straightforward to determine, as we demonstrate in the Proposition A.1. The optimal $\operatorname{VaR}_{\alpha}^{*}\left(S_{n}^{n}\right)$, on the other hand, is more complicated and it requires us to consider several additional subcases, as we will later elaborate.

Before presenting some key results, it is useful to give the following explicit expressions for $\operatorname{VaR}_{\alpha}(\boldsymbol{c}, \boldsymbol{d})$ pertaining to a given confidence level $1-\alpha$ with $0<\alpha<S_{X}(0)$ :

(i) When $\delta_{\alpha} \leqslant d_{n, 1}$, that is, $\left(d_{n, 1}, \ldots, d_{n, n}\right) \in D_{n}^{0}$,

$$
\operatorname{VaR}_{\alpha}(\boldsymbol{c}, \boldsymbol{d})=\delta_{\alpha}+(1+\theta)\left\{\sum_{j=1}^{n} c_{n, j} \int_{d_{n, j}}^{\infty} S_{X}(x) d x\right\} ;
$$

(ii) When $d_{n, i} \leqslant \delta_{\alpha} \leqslant d_{n, i+1}$, that is, $\left(d_{n, 1}, \ldots, d_{n, n}\right) \in D_{n}^{i}$, and for $i=1, \ldots, n-1$,

$$
\operatorname{VaR}_{\alpha}(\boldsymbol{c}, \boldsymbol{d})=\delta_{\alpha}+\sum_{j=1}^{i} c_{n, j}\left[d_{n, j}-\delta_{\alpha}\right]+(1+\theta)\left\{\sum_{j=1}^{n} c_{n, j} \int_{d_{n, j}}^{\infty} S_{X}(x) d x\right\} ;
$$

(iii) When $d_{n, n} \leqslant \delta_{\alpha}$, that is, $\left(d_{n, 1}, \ldots, d_{n, n}\right) \in D_{n}^{n}$,

$$
\operatorname{VaR}_{\alpha}(\boldsymbol{c}, \boldsymbol{d})=\delta_{\alpha}+\sum_{j=1}^{n} c_{n, j} \kappa\left(d_{n, j}\right) .
$$

We now present the following proposition, which summarizes the optimality for the first $n$ partitions of $S_{n}$ :

\section{Proposition A.1}

(a) Consider minimizing $\operatorname{VaR}_{\alpha}(\boldsymbol{c}, \boldsymbol{d})$ with feasible set $S_{n}^{0}, \boldsymbol{c}^{*}=\mathbf{0}$ is one optimal solution of $\operatorname{VaR}_{\alpha}^{*}\left(S_{n}^{0}\right)$ with optimal minimum value $\operatorname{VaR}_{\alpha}^{*}\left(S_{n}^{0}\right)=\delta_{\alpha}$.

(b) Consider minimizing $\operatorname{VaR}_{\alpha}(\boldsymbol{c}, \boldsymbol{d})$ with feasible set $S_{n}^{i}, i=1,2, \ldots, n-1$, the optimal solutions $\left(c^{*}, d^{*}\right)$ satisfy either $d_{n, j}^{*} \rightarrow \infty$ for $j=i+1, \ldots, n$ or equivalently $c_{n, j}^{*}=0$ for $j=i+1, \ldots, n$.

Proof The proof is trivial by the expressions of $\operatorname{VaR}_{\alpha}(\boldsymbol{c}, \boldsymbol{d})$ in (A.8) and (A.9).

The only task remaining is to consider the optimal solution on the final partition $S_{n}^{n}$. As alluded earlier that the optimality associated with $S_{n}^{n}$ is more 
complicated to analyse. It entails us to sub-partitioning the feasible set $S_{n}^{n}$ into a few more subcases depending on the relative magnitude of $\alpha$ and $\rho$, the sign of $\kappa\left(\delta_{\rho}\right)$, and whether $\beta\left(\delta_{\rho}\right)$ is greater or smaller than one. More specifically, there are seven subcases in total to be considered and these are listed below. A flowchart of these subcases is depicted in Figure A.1. For ease of referencing, we also include the respective propositions that deal with each of these subcases.

Case (i) $\alpha \geqslant \rho$, see Proposition A.2(a).

Case (ii) $\quad \alpha<\rho, \kappa\left(\delta_{\rho}\right)>0$, see Proposition A.2(b).

Case (iii) $\alpha<\rho, \kappa\left(\delta_{\rho}\right)=0$, see Proposition A.3(a).

Case (iv) $\alpha<\rho, \kappa\left(\delta_{\rho}\right)<0$, and $\beta\left(\delta_{\rho}\right)>1$, see Proposition A.3(b).

Case (v) $\quad \alpha<\rho, \kappa\left(\delta_{\rho}\right)<0, \beta\left(\delta_{\rho}\right) \leqslant 1$, and $\lambda\left(\delta_{\rho}\right) \geqslant 0$, see Proposition A.4(a).

Case (vi) $\alpha<\rho, \kappa\left(\delta_{\rho}\right)<0, \beta\left(\delta_{\rho}\right) \leqslant 1, \lambda\left(\delta_{\rho}\right)<0$, and $\beta\left(d_{o}\right) \leqslant 1$, see Proposition A.4b(i).

Case (vii) $\alpha<\rho, \kappa\left(\delta_{\rho}\right)<0, \beta\left(\delta_{\rho}\right) \leqslant 1, \lambda\left(\delta_{\rho}\right)<0$, and $\beta\left(d_{o}\right)>1$, see Proposition A. $4 \mathrm{~b}$ (ii).

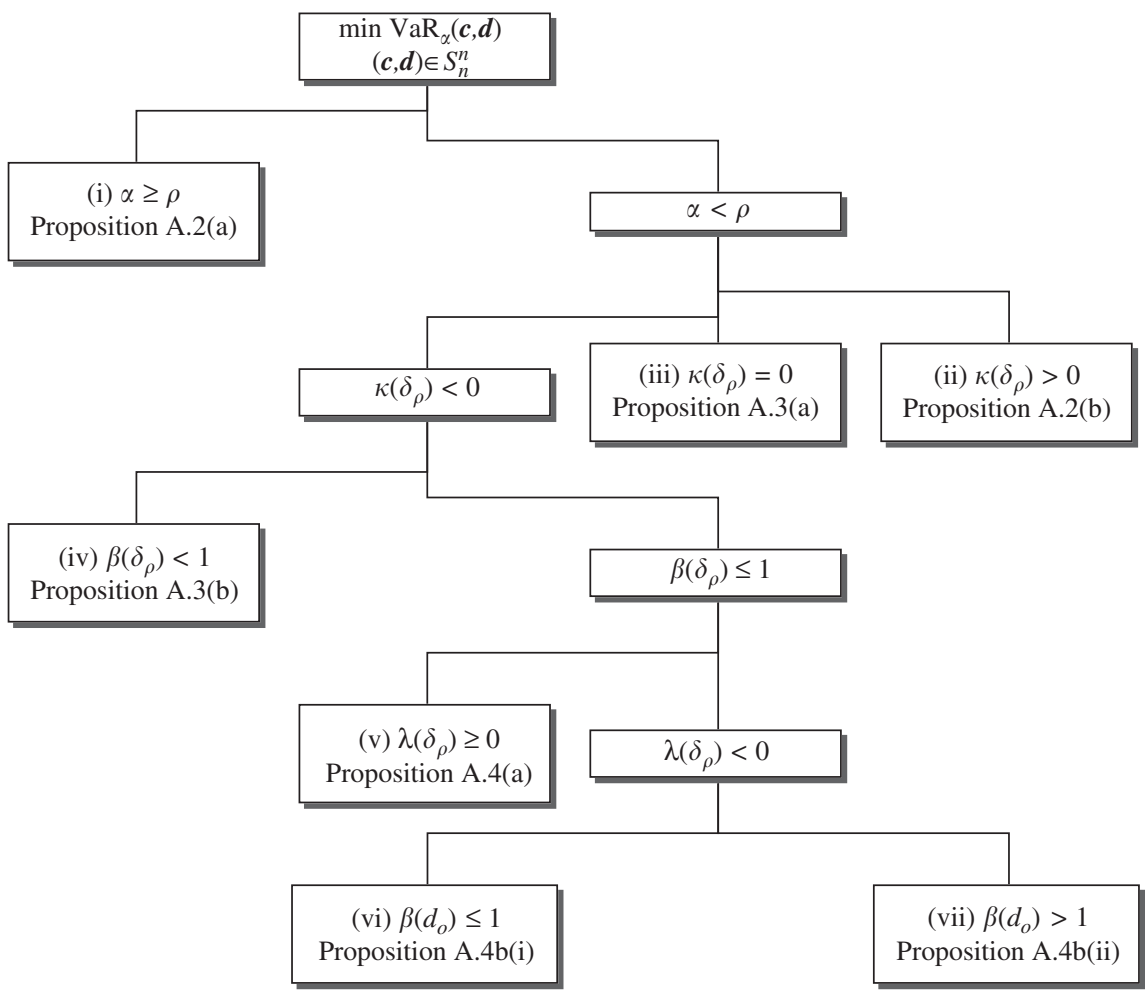

Figure A.1. Subcases of $S_{n}^{n}$. 
We first present the trivial cases where it is never optimal for the insurer to reinsure its risk. These correspond to cases (a) $\alpha \geqslant \rho$ and (b) $\alpha<\rho$ with $\kappa\left(\delta_{\rho}\right)>0$, as we show in the following proposition:

Proposition A.2 Consider minimizing $\operatorname{VaR}_{\alpha}(\boldsymbol{c}, \boldsymbol{d})$ with feasible set $S_{n}^{n}$. When (a) $\alpha \geqslant \rho$ or (b) $\alpha<\rho$ and $\kappa\left(\delta_{\rho}\right)>0, c^{*}=\mathbf{0}$ is one solution with $\operatorname{VaR}_{\alpha}^{*}\left(S_{n}^{n}\right)=\delta_{\alpha}$.

Proof The condition $\alpha \geqslant \rho$ implies $\delta_{\rho} \geqslant \delta_{\alpha}$ and using Lemma B.1(a) (see Appendix B), we have

$$
\min _{d \in\left[0, \delta_{\alpha}\right]} \kappa(d)=u\left(\delta_{\alpha}\right)=(1+\theta) \int_{\delta_{\alpha}}^{\infty} S_{X}(x) d x>0 .
$$

Thus, $\kappa(d)>0$ for all $d \in\left[0, \delta_{\alpha}\right]$. Since on $S_{n}^{n}$ we have $d_{n, j} \leqslant \delta_{\alpha}$ for $j=1,2, \ldots, n$, it follows from (A.10) that $\operatorname{VaR}_{\alpha}(\boldsymbol{c}, \boldsymbol{d})$ attains its minimum value when $\boldsymbol{c}=\mathbf{0}$. Hence $\operatorname{VaR}_{\alpha}^{*}\left(S_{n}^{n}\right)=\delta_{\alpha}$. The second case with $\alpha<\rho$ and $\kappa\left(\delta_{\rho}\right)>0$ can be proved similarly since from Lemma B.1(a), we have

$$
\min _{d \in\left[0, \delta_{\alpha}\right]} \kappa(d)=\kappa\left(\delta_{\rho}\right)>0 .
$$

In order to discuss the optimal solutions corresponding to Cases (iii) and (iv), it is essential to introduce the following sets:

$$
\begin{aligned}
& T_{n}^{n}:=\left\{(\boldsymbol{c}, \boldsymbol{d}):(\boldsymbol{c}, \boldsymbol{d}) \in S_{n}^{n}, d_{n, 1} \geqslant \delta_{\rho}\right\}, \\
& T_{n}^{\geqslant}:=\left\{(\boldsymbol{c}, \boldsymbol{d}):(\boldsymbol{c}, \boldsymbol{d}) \in T_{n}^{n}, \sum_{j=1}^{n} c_{n, j} \geqslant \beta\left(\delta_{\rho}\right)\right\}, \\
& T_{n}^{\leqslant}:=\left\{(\boldsymbol{c}, \boldsymbol{d}):(\boldsymbol{c}, \boldsymbol{d}) \in T_{n}^{n}, \sum_{j=1}^{n} c_{n, j} \leqslant \beta\left(\delta_{\rho}\right)\right\} .
\end{aligned}
$$

Recall that $\beta\left(\delta_{\rho}\right)$ was defined through (14) and (15), and that $T_{n}^{\geqslant}$is an empty set when $\beta\left(\delta_{\rho}\right)>1$. Furthermore, $T_{n}^{\geqslant}$and $T_{n}^{\leqslant}$are the partitioned sets of $T_{n}^{n}$, that is $T_{n}^{n}=T_{n}^{\geqslant} \bigcup T_{n}^{\leqslant}$. Exploiting the partitioning, Lemma B.2 in Appendix B establishes the following relation for $\alpha<\rho$ :

$$
\operatorname{VaR}_{\alpha}^{*}\left(S_{n}^{n}\right)=\operatorname{VaR}_{\alpha}^{*}\left(T_{n}^{n}\right)
$$

The above result is useful in the sense that under the prescribed condition, it is sufficient to focus the optimality on $T_{n}^{n}$ for identifying solution on $S_{n}^{n}$. This 
relation is used explicitly in deriving the optimal solutions for cases (iii) and (iv), as we show in the following proposition:

Proposition A.3 Consider minimizing $\operatorname{VaR}_{\alpha}(\boldsymbol{c}, \boldsymbol{d})$ with feasible set $S_{n}^{n}$.

(a) When $\alpha<\rho$ and $\kappa\left(\delta_{\rho}\right)=0,\left(c^{*}, \delta_{\rho}\right)$ such that $0 \leqslant c^{*} \leqslant \min \left\{\beta\left(\delta_{\rho}\right), 1\right\}$ is one solution with $\operatorname{VaR}_{\alpha}^{*}\left(S_{n}^{n}\right)=\delta_{\alpha}$.

(b) When $\alpha<\rho, \kappa\left(\delta_{\rho}\right)<0$ and $\beta\left(\delta_{\rho}\right)>1, \quad\left(1, \delta_{\rho}\right)$ is one solution with $\operatorname{VaR}_{\alpha}^{*}\left(S_{n}^{n}\right)=\delta_{\alpha}+\kappa\left(\delta_{\rho}\right)<\delta_{\alpha}$.

Proof Lemma B.2 in Appendix B shows that $\operatorname{VaR}_{\alpha}^{*}\left(S_{n}^{n}\right)=\operatorname{VaR}_{\alpha}^{*}\left(T_{n}^{n}\right)$ and for any vectors $(c, d) \in T_{n}^{n}$,

$$
\operatorname{VaR}_{\alpha}(\boldsymbol{c}, \boldsymbol{d})=\delta_{\alpha}+\sum_{j=1}^{n} c_{n, j} \kappa\left(d_{n, j}\right) \geqslant \delta_{\alpha}+\kappa\left(\delta_{\rho}\right) \sum_{j=1}^{n} c_{n, j}
$$

(a) When $\alpha<\rho$ and $\kappa\left(\delta_{\rho}\right)=0$, (A.12) becomes $\operatorname{VaR}_{\alpha}(\boldsymbol{c}, \boldsymbol{d}) \geqslant \delta_{\alpha}$. The lower bound corresponds to $\operatorname{VaR}_{\alpha}\left(c^{*}, \delta_{\rho}\right)$ with $c^{*}$ as defined in the proposition, and the upper bound restriction on $c^{*}$ ensures that $\left(c^{*}, \delta_{\rho}\right) \in T_{n}^{n}$; hence the results follow.

(b) To establish the optimality for $\alpha<\rho, \kappa\left(\delta_{\rho}\right)<0$ and $\beta\left(\delta_{\rho}\right)>1$, first recall that $T_{n}^{n}=T_{n}^{\geqslant} \bigcup T_{n}^{\leqslant}$and $T_{n}^{\geqslant}$is an empty set when $\beta\left(\delta_{\rho}\right)>1$. Hence we only need to show that $\left(1, \delta_{\rho}\right)$ is optimal over $T_{n}^{\leqslant}$. It follows from the condition $\kappa\left(\delta_{\rho}\right)<0$ that the lower bound (A.12) can further be reduced to $\delta_{\alpha}+\kappa\left(\delta_{\rho}\right)$, which is equal to $\left(1, \delta_{\rho}\right)$. Since $\beta\left(\delta_{\rho}\right)>1,\left(1, \delta_{\rho}\right)$ is obviously within $T_{n}^{\leqslant}$and hence we obtain the required results.

To analyse the remaining three cases pertaining to conditions $\alpha<\rho, \kappa\left(\delta_{\rho}\right)<0$, and $\beta\left(\delta_{\rho}\right) \leqslant 1$, we again employ the same approach as above except that in these cases, we consider the following set:

$$
V:=\left\{(c, d) \in \mathbb{R}: \delta_{\rho} \leqslant d \leqslant \delta_{\alpha}, c \int_{d}^{\infty} S_{X}(x) d x=B, 0 \leqslant c \leqslant 1\right\} .
$$

Note that $V$ is well defined since $\delta_{\rho} \leqslant \delta_{\alpha}$ due to the condition $\alpha<\rho$. Lemma B.5 similarly justifies that

$$
\operatorname{VaR}_{\alpha}^{*}\left(S_{n}^{n}\right)=\operatorname{VaR}_{\alpha}^{*}(V)
$$


and therefore it is sufficient to just focus on the set $V$ for the optimality on $S_{n}^{n}$. This is demonstrated in the following proposition which completes the analysis for Cases (v), (vi), and (vii):

Proposition A.4 Suppose $\alpha<\rho, \kappa\left(\delta_{\rho}\right)<0$, and $\beta\left(\delta_{\rho}\right) \leqslant 1$, and consider minimizing $\operatorname{VaR}_{\alpha}(c, d)$ with feasible set $V$.

(a) If $\lambda\left(\delta_{\rho}\right) \geqslant 0$, then $\left(\beta\left(\delta_{\rho}\right), \delta_{\rho}\right)$ is one solution with $\operatorname{VaR}_{\alpha}^{*}(V)=$ $\delta_{\alpha}+\beta\left(\delta_{\rho}\right) \cdot \kappa\left(\delta_{\rho}\right)<\delta_{\alpha}$.

(b) Suppose $\lambda\left(\delta_{\rho}\right)<0$.

(b.a) If $\beta\left(d_{o}\right) \leqslant 1$, then $\left(\beta\left(d_{o}\right), d_{o}\right)$ is one solution with $\operatorname{VaR}_{\alpha}^{*}(V)=\delta_{\alpha}-B[(1) /$ $\left.\left(S_{X}\left(d_{o}\right)\right)-(1) /(\rho)\right]<\delta_{\alpha}$, where as defined in Lemma $1(\mathrm{~b}), d_{o} \in\left(\delta_{\rho}, \delta_{\alpha}\right)$ satisfies $\lambda\left(d_{o}\right)=0$.

(b.b) If $\beta\left(d_{o}\right)>1$, then $\left(1, d_{B}\right)$ is one solution with $\operatorname{VaR}_{\alpha}^{*}(V)=\delta_{\alpha}+u\left(d_{B}\right)<$ $\delta_{\alpha}$, where $d_{B}$ is defined in (18).

Proof For any coefficient pair $(c, d) \in V$, it follows from (A.10) that

$$
\begin{aligned}
\operatorname{VaR}_{\alpha}(c, d) & =\delta_{\alpha}+c\left(d-\delta_{\alpha}\right)+(1+\theta) c \int_{d}^{\infty} S_{X}(x) d x \\
& =\delta_{\alpha}+(1+\theta) B+\frac{d-\delta_{\alpha}}{\int_{d}^{\infty} S_{X}(x) d x} B .
\end{aligned}
$$

Because of the condition $c \int_{d}^{\infty} S_{X}(x) d x=B$, the coefficient $c$ uniquely determines $d$ (and vice-versa) and hence $\operatorname{VaR}_{\alpha}(c, d)$ can be regarded as either a function of $c$ or $d$. For the sake of our analysis, we will express $\operatorname{VaR}_{\alpha}(c, d)$ as a function as we have shown in (A.15) and we will denote it by $\Gamma(d)$. The derivative of $\Gamma(d)$ with respect to $d$ is

$$
\Gamma^{\prime}(d)=\frac{\lambda(d)}{\left[\int_{d}^{\infty} S_{X}(x) d x\right]^{2}} B .
$$

(a) When $\lambda\left(\delta_{\rho}\right) \geqslant 0$, then $\Gamma^{\prime}(d)>0$ on $\left(\delta_{\rho}, \delta_{\alpha}\right]$ since $\lambda(d)$ is strictly increasing on $\left[0, \delta_{\alpha}\right]$ by Lemma B.1(b). Hence $\Gamma(d)$ is strictly increasing on $\left[\delta_{\rho}, \delta_{\alpha}\right]$ and it attains its minimum value at $d=\delta_{\rho}$. This implies that $\operatorname{VaR}_{\alpha}^{*}(V)=$ $\delta_{\alpha}+\beta\left(\delta_{\rho}\right) \cdot \kappa\left(\delta_{\rho}\right)<\delta_{\alpha}$. The last strict inequality is due to $\kappa\left(\delta_{\rho}\right)<0$.

(b) Under the assumption $\lambda\left(\delta_{\rho}\right)<0$, Lemma B.1(b) assures that $\Gamma^{\prime}(d)<0$ for $d \in\left[\delta_{\rho}, d_{o}\right)$, and $\Gamma^{\prime}(d)>0$ for $d \in\left(d_{o}, \delta_{\alpha}\right]$. This in turn implies $\Gamma(d)$ is strictly decreasing on $\left[\delta_{\rho}, d_{o}\right]$ while strictly increasing on $\left[d_{o}, \delta_{\alpha}\right]$, and it attains the minimum value at $d=d_{o}$. 
Recall that any coefficient pair $(c, d) \in V$ must satisfy the constraint $c \int_{d}^{\infty} S_{X}(x) d x=B$ and $0 \leqslant c \leqslant 1$. Hence when $\beta\left(d_{o}\right) \leqslant 1,\left(\beta\left(d_{o}\right), d_{o}\right)$ is one optimal solution on $V$. Moreover because the minimum value of $\Gamma(d)$ is attained at $d=d_{o}$, we have

$$
\begin{aligned}
\operatorname{VaR}_{\alpha}^{*}(V) & =\Gamma\left(d_{o}\right)<\Gamma\left(\delta_{\rho}\right) \\
& =\delta_{\alpha}+\beta\left(\delta_{\rho}\right) \cdot \kappa\left(\delta_{\rho}\right)<\delta_{\alpha}
\end{aligned}
$$

and $\operatorname{VaR}_{\alpha}^{*}(V)$ is easily shown to be

$$
\begin{aligned}
\operatorname{VaR}_{\alpha}^{*}(V) & =\delta_{\alpha}+(1+\theta) B+B \cdot \frac{d_{o}-\delta_{\alpha}}{\int_{d_{o}}^{\infty} S_{X}(x) d x} \\
& =\delta_{\alpha}-B\left[\frac{1}{S_{X}\left(d_{o}\right)}-\frac{1}{\rho}\right]
\end{aligned}
$$

as claimed in Part (i).

(c) For Part (ii) with the condition $\beta\left(d_{o}\right)>1$, first note that $\left(\beta\left(d_{o}\right), d_{o}\right)$ is no longer in $V$. Second, the property that $\Gamma(d)$ is strictly decreasing on $\left[\delta_{\rho}, d_{o}\right]$ while strictly increasing on $\left[d_{o}, \delta_{\alpha}\right]$ ensures that the minimum of $\operatorname{VaR}_{\alpha}(c, d)$ over $V$ while subject to the constraint $0<c \leqslant 1$ must occur at $d_{B}$ that satisfies (18). Finally, it is also obvious that $\operatorname{VaR}_{\alpha}^{*}(V)=\Gamma\left(d_{B}\right) \leqslant \Gamma\left(\delta_{\rho}\right)<\delta_{\alpha}$ and this completes the proof.

Now that we have analysed the optimal solutions to all the partitions $S_{n}^{i}$, $i=0, \ldots, n$, we are ready to present the optimal solution to (A.6) or equivalently (A.2). Recall that the global solution corresponds to the partition that satisfies (A.7). Proposition A.1(a) immediately establishes that the minimum VaR cannot be greater than $\delta_{\alpha}$. This result, together with Proposition A.1(b) and equation (A.9), further imply that the minimum VaR cannot fall into partitions $S_{n}^{i}, i=1, \ldots, n-1$. Consequently, the least value $\operatorname{VaR}_{\alpha}^{*}\left(S_{n}\right)$, with optimal $\left(c^{*}, d^{*}\right)$, can only derive from either partition $S_{n}^{0}$ or partition $S_{n}^{n}$. More specifically, when $\alpha \geqslant \rho$ or $\alpha<\rho$ and $\kappa\left(\delta_{\rho}\right) \geqslant 0$, then we have $\operatorname{VaR}_{\alpha}^{*}\left(S_{n}\right)=\delta_{\alpha}$. Furthermore, when (i) $\alpha \geqslant \rho$ and (ii) $\alpha<\rho$ and $k\left(\delta_{\rho}\right)>0$, Proposition A.2(a) and Proposition A.2(b), respectively, confirm that it is never optimal to cede losses to a reinsurer since $c^{*}=0$ is the optimal coefficient. The case with $\alpha<\rho$ and $\kappa\left(\delta_{\rho}\right)=0$ (i.e. Case (iii)) illustrates an interesting situation that it can be both optimal to reinsure and not to reinsure losses since the optimal coefficients are $\left(c^{*}, \delta_{\rho}\right)$, for $0 \leqslant c^{*} \leqslant \min \left\{\beta\left(\delta_{\rho}\right), 1\right\}$ (see Proposition A.3(a)). When $\alpha<\rho$ and $\kappa\left(\delta_{\rho}\right)<0$, Proposition A.3(b) and Proposition A.4 assure that $\operatorname{VaR}_{\alpha}^{*}\left(S_{n}\right)<\delta_{\alpha}$ with possible optimal coefficients $\left(1, \delta_{\rho}\right),\left(\beta\left(\delta_{\rho}\right), \delta_{\rho}\right),\left(\beta\left(d_{o}\right), d_{o}\right)$, or $\left(1, d_{B}\right)$. 
Table A1 Optimal solutions to the approximation models (A.6)

\begin{tabular}{llllc}
\hline Case & Conditions & $\left(\boldsymbol{c}^{*}, \boldsymbol{d}^{*}\right)$ & VaR & Proposition \\
\hline (i) & $\alpha \geqslant \rho$ & $(0, d)$, any $d \geqslant 0$ & $\delta_{\alpha}$ & A.2(a) \\
(ii) & $\alpha<\rho, \kappa\left(\delta_{\rho}\right)>0$ & $(0, d)$, any $d \geqslant 0$ & $\delta_{\alpha}$ & A.2(b) \\
(iii) & $\alpha<\rho, \kappa\left(\delta_{\rho}\right)=0$ & $\left(c^{*}, \delta_{\rho}\right)$, for & $\delta_{\alpha}$ & A.3(a) \\
& & $0 \leqslant c^{*} \leqslant \min \left\{\beta\left(\delta_{\rho}\right), 1\right\}$ & & \\
(iv) $\quad \alpha<\rho, \kappa\left(\delta_{\rho}\right)<0, \beta\left(\delta_{\rho}\right)>1$ & $\left(1, \delta_{\rho}\right)$ & $\delta_{\alpha}+\kappa\left(\delta_{\rho}\right)$ & A.3(b) \\
(v) $\quad \alpha<\rho, \kappa\left(\delta_{\rho}\right)<0$, & $\left(\beta\left(\delta_{\rho}\right), \delta_{\rho}\right)$ & $\delta_{\alpha}+\beta\left(\delta_{\rho}\right) \kappa\left(\delta_{\rho}\right)$ & A.4(a) \\
& $\beta\left(\delta_{\rho}\right) \leqslant 1, \lambda\left(\delta_{\rho}\right) \geqslant 0$ & $\left(\beta\left(d_{o}\right), d_{o}\right)$ & $\delta_{\alpha}-B\left((1) /\left(S_{X}\left(d_{o}\right)\right)-\right.$ & A.4b(i) \\
(vi) & $\alpha<\rho, \kappa\left(\delta_{\rho}\right)<0$, & $(1) /(\rho))$ & \\
& $\beta\left(\delta_{\rho}\right) \leqslant 1, \lambda\left(\delta_{\rho}\right)<0, \beta\left(d_{o}\right) \leqslant 1$ & & $\delta_{\alpha}+u\left(d_{B}\right)$ & A.4b(ii) \\
(vii) & $\alpha<\rho, \kappa\left(\delta_{\rho}\right)<0$, & $\left(1, d_{B}\right)$ & & \\
& $\beta\left(\delta_{\rho}\right) \leqslant 1, \lambda\left(\delta_{\rho}\right)<0, \beta\left(d_{o}\right)>1$ & & &
\end{tabular}

Table A1 provides a summary of all the possible $\operatorname{VaR}_{\alpha}^{*}\left(S_{n}\right)$ together with their optimal coefficients $\left(c^{*}, d^{*}\right)$. A notable feature about these results is that even though we begin our analysis by optimally seeking $n$-dimensional vectors $\left(c^{*}, d^{*}\right)$ to the optimization problem (A.6) for a given $n$, it turns out that the derived optimal solutions are independent of $n$. This implies that by (A.1), the optimal solutions to (A.2) are also independent of $n$ and these are reported in the column of $\mu^{*}$ in Table 1 .

We conclude the appendix by demonstrating that the solutions derived for model (A.2) are also the solutions for model (13). This is stated formally in the following proposition:

Proposition A.5 The solutions $\mu^{*}$ summarized in Table 1 (with optimal $\mathrm{VaR}_{\alpha}^{*}$ ) are also the optimal solutions to the optimization problem (13).

Proof Recall that $\mu^{*}$ in Table 1 solves model (A.2) for all $n=1,2, \ldots$ Let $\mu$ be any positive measure from the feasible set of problem (13), that is, $\mu \in \mathcal{M}^{+}$and $\int \gamma(t) d \mu(t) \leqslant B$. We need to show $\operatorname{VaR}_{\alpha}\left(\mu^{*}\right) \leqslant \operatorname{VaR}_{\alpha}(\mu)$. Before we proceed, it is helpful to recall the notation $\phi(t)=\left(\operatorname{VaR}_{\alpha}(X)-t\right)_{+}$and $\psi(t)=E\left[(X-t)_{+}\right]$.

By Lemma B.6, there exists a sequence of measures $\left\{\mu_{n}, n=1,2, \ldots\right\}$ in $\mathcal{M}_{n}^{+}$ such that $\int(x-t)_{+} d \mu_{n}$ converges pointwisely to $\int(x-t)_{+} d \mu$ from below. This fact has two crucial implications which are important for completing the proof. On the one hand, we have $\int(x-t)_{+} d \mu_{n} \leqslant B$ and hence $\int \psi(t) d \mu_{n} \leqslant B$ by the Fubini theory for $n=1,2, \ldots$ This implies that $\mu_{n}$ belongs to the feasible set of problem (A.2) for $n=1,2, \ldots$, and consequently we have

$$
\operatorname{VaR}_{\alpha}\left(\mu^{*}\right) \leqslant \operatorname{VaR}_{\alpha}\left(\mu_{n}\right) \text { for } n=1,2, \ldots
$$


On the other hand, by Fubini's Theorem and Lemma B.6, we have

and

$$
\begin{aligned}
\int \phi(t) d \mu_{n} & =\int\left[\operatorname{VaR}_{\alpha}(X)-t\right]_{+} d \mu_{n} \\
& \rightarrow \int\left[\operatorname{VaR}_{\alpha}(X)-t\right]_{+} d \mu=\int \phi(t) d \mu_{n}
\end{aligned}
$$

$$
\begin{aligned}
\int \psi(t) d \mu_{n} & =\int E(X-t)_{+} d \mu_{n}=E\left[\int(X-t)_{+} d \mu_{n}\right] \\
& \rightarrow E\left[\int(X-t)_{+} d \mu\right]=\int E(X-t)_{+} d \mu=\int \psi(t) d \mu
\end{aligned}
$$

where the first convergence result is due to Lemma B.6 and the second convergence result is the combination of Lemma B.6 and the monotonic convergence theorem. These results in turn lead to

$$
\begin{aligned}
\operatorname{VaR}_{\alpha}\left(\mu_{n}\right)= & \operatorname{VaR}_{\alpha}(X)-\int[\phi(t)-(1+\theta) \psi(t)] d \mu_{n} \\
& \rightarrow \operatorname{VaR}_{\alpha}(X)-\int[\phi(t)-(1+\theta) \psi(t)] d \mu \\
= & \operatorname{VaR}_{\alpha}(\mu) .
\end{aligned}
$$

Finally, by combining (A.19) and (A.20), we immediately have $\operatorname{VaR}_{\alpha}\left(\mu^{*}\right)$ $\leqslant \operatorname{VaR}_{\alpha}(\mu)$ and this completes the proof.

\section{Appendix B}

Some lemmas

This appendix collects the lemmas that are relevant to proving the main results presented in Appendix A.

\section{Lemma B.1}

(a) The continuous function $\kappa(d)$ defined in (16) is convex for $d \geqslant 0$. Moreover, if $\rho<S_{X}(0)$, then $\kappa(d)$ is decreasing on $\left[0, \delta_{\rho}\right]$ while increasing on $\left[\delta_{\rho}, \infty\right)$ and satisfies

$$
\begin{gathered}
\min _{0 \leqslant d \leqslant a}\{\kappa(d)\}=\kappa(a) \text { for } 0 \leqslant a \leqslant \delta_{\rho}, \\
\min _{0 \leqslant d \leqslant a}\{\kappa(d)\}=\kappa\left(\delta_{\rho}\right) \text { for } \delta_{\rho} \leqslant a ;
\end{gathered}
$$


(b) if $\rho \geqslant S_{X}(0)$, then $\kappa(d)$ is increasing on $(0, \infty)$ and satisfies

$$
\min _{0 \leqslant d \leqslant a}\{\kappa(d)\}=\kappa(0) \text { for } a \geqslant 0 .
$$

(c) The continuous function $\lambda(d)$ defined in (17) is strictly increasing on $\left[0, \delta_{\alpha}\right]$. Moreover, when $\alpha<\rho$, and $\lambda\left(\delta_{\rho}\right)<0$, there exists a unique root $d_{o}$ to the equation $\lambda(d)=0$ on $\left(\delta_{\rho}, \delta_{\alpha}\right)$.

\section{Proof}

(a) First note that the derivatives of $\kappa(d), \kappa^{\prime}(d)=1-(1+\theta) S_{X}(d)$, and $\kappa^{\prime \prime}(d)=(1+\theta) f_{X}(d) \geqslant 0$ for $d \geqslant 0$. Hence $\kappa(d)$ is convex on $(0, \infty)$. Moreover, we can easily verify that if $\rho<S_{X}(0)$, then $\kappa^{\prime}(d)<0$ for $0<d<\delta_{\rho}$ and $\kappa^{\prime}(d)>0$ for $d>\delta_{\rho}$, and that $\kappa^{\prime}(d)>0$ for $d>0$ if $\rho \geqslant S_{X}(0)$. This justifies (B.1)-(B.3).

(b) It follows from (17) that $\lambda^{\prime}(d)=\left[\delta_{\alpha}-d\right] f_{X}(d)$. By the assumption that $X$ has strictly increasing distribution, we have $f_{X}(d)>0$ for $d \geqslant 0$ so that $\lambda^{\prime}(d)>0$ for $d \in\left[0, \delta_{\alpha}\right)$. As a result, the function $\lambda(d)$ is strictly increasing on $\left[0, \delta_{\alpha}\right]$. Moreover, we have $\lambda\left(\delta_{\alpha}\right)=\int_{\delta_{\alpha}}^{\infty} S_{X}(x) d x>0$, and $\delta_{\rho}<\delta_{\alpha}$ as $\alpha<\rho$. Hence, there must exist a unique root $d_{o}$ to the equation $\lambda(d)=0$ on $\left(\delta_{\rho}, \delta_{\alpha}\right)$ as we have $\lambda\left(\delta_{\rho}\right)<0$.

Lemma B.2 If $\alpha<\rho$, then $\operatorname{VaR}_{\alpha}^{*}\left(S_{n}^{n}\right)=\operatorname{VaR}_{\alpha}^{*}\left(T_{n}^{n}\right)$; that is (A.11) holds.

Proof If $\rho \geqslant S_{X}(0)$, then (A.11) holds trivially since $\delta_{\rho}=0$ so that $S_{n}^{n}=T_{n}^{n}$. Suppose $\rho<S_{X}(0)$. Let $(\boldsymbol{c}, \boldsymbol{d})$ be any vectors in $S_{n}^{n}$ satisfying $d_{n, j}<\delta_{\rho}, j=1,2, \ldots, i$, for a fixed $i \in\{1,2, \ldots, n\}$ and let $\left(\boldsymbol{c}, \boldsymbol{d}^{\prime}\right)$ be the corresponding vectors constructed from $(\boldsymbol{c}, \boldsymbol{d})$ by merely replacing $d_{n, j}$ with $\delta_{\rho}$ for all $j=1,2, \ldots, i$. Since

$$
\sum_{j=1}^{i} c_{n, j} \int_{\delta_{\rho}}^{\infty} S_{X}(x) d x+\sum_{j=i+1}^{n} c_{n, j} \int_{d_{n, j}}^{\infty} S_{X}(x) d x \leqslant \sum_{j=1}^{n} c_{n, j} \int_{d_{n, j}}^{\infty} S_{X}(x) d x \leqslant B,
$$

$\left(\boldsymbol{c}, \boldsymbol{d}^{\prime}\right)$ is also in $S_{n}^{n}$ and hence in $T_{n}^{n}$. Moreover,

$$
\begin{aligned}
\operatorname{VaR}_{\alpha}(\boldsymbol{c}, \boldsymbol{d})= & \delta_{\alpha}+\sum_{j=1}^{n} c_{n, j} \kappa\left(d_{n, j}\right) \\
& \geqslant \delta_{\alpha}+\sum_{j=1}^{i} c_{n, j} \kappa\left(\delta_{\rho}\right)+\sum_{j=i+1}^{n} c_{n, j} \kappa\left(d_{n, j}\right) \\
& =\operatorname{VaR}_{\alpha}\left(c, d^{\prime}\right),
\end{aligned}
$$


since the minimum value of the function $\kappa(x)$ is $\kappa\left(\delta_{\rho}\right)$ by part (a) of Lemma 4 and this completes the proof.

Lemma B.3 If $\alpha<\rho, \kappa\left(\delta_{\rho}\right)<0$ and $\beta\left(\delta_{\rho}\right) \leqslant 1$, then the following relation holds

$$
\operatorname{VaR}_{\alpha}^{*}\left(T_{n}^{\geqslant}\right)=\operatorname{VaR}_{\alpha}^{*}(V) .
$$

Proof We prove (B.5) via the following two-step procedure. First we establish

$$
\operatorname{VaR}_{\alpha}^{*}\left(T_{n}^{\geqslant}\right)=\operatorname{VaR}_{\alpha}^{*}(U),
$$

where $U$ is a set defined as

$$
U=\left\{(\boldsymbol{c}, \boldsymbol{d}):(\boldsymbol{c}, \boldsymbol{d}) \in T_{n}^{\geqslant}, \sum_{j=1}^{n} c_{n, j} \int_{d_{n, j}}^{\infty} S_{X}(x) d x=B\right\}
$$

Then we show that

$$
\operatorname{VaR}_{\alpha}^{*}(U)=\operatorname{VaR}_{\alpha}^{*}(V)
$$

to complete the proof.

Now for the first part of the proof of showing (B.6), we demonstrate the following two inequalities:

$$
\begin{gathered}
\operatorname{VaR}_{\alpha}^{*}\left(T_{n}^{\geqslant}\right) \leqslant \operatorname{VaR}_{\alpha}^{*}(U), \\
\operatorname{VaR}_{\alpha}^{*}\left(T_{n}^{\geqslant}\right) \geqslant \operatorname{VaR}_{\alpha}^{*}(U) .
\end{gathered}
$$

The first inequality is straightforward as it follows immediately from the definition that $U \subset T_{n}^{\geqslant}$. To justify the second inequality, first note that for any $(\boldsymbol{c}, \boldsymbol{d}) \in T_{n}^{\geqslant}$, we have $d_{n, j} \geqslant \delta_{\rho}$ for $j=1,2, \ldots, n$ and that both

$$
\sum_{j=1}^{n} c_{n, j} \int_{\delta_{\rho}}^{\infty} S_{X}(x) d x \geqslant B
$$


and

$$
\sum_{j=1}^{n} c_{n, j} \int_{d_{n, j}}^{\infty} S_{X}(x) d x \leqslant B
$$

hold simultaneously. The above two inequalities follow from the constraint on the sets $T_{n}^{\geqslant}$and $S_{n}^{n}$, respectively. Moreover, $\sum_{j=1}^{n} c_{n, j} \int_{d_{n, j}}^{\infty} S_{X}(x) d x$ is continuous in $d_{n, j}$ for $j=1,2, \ldots, n$, since we assume $X$ has a continuous distribution function on $(0, \infty)$. Thus, for any $(\boldsymbol{c}, \boldsymbol{d}) \in T_{n}^{\geqslant}$satisfying (12), there exist constants $\delta_{\rho} \leqslant d_{n, j}^{\prime} \leqslant d_{n, j}, j=1,2, \ldots, n$ such that $\sum_{j=1}^{n} c_{n, j} \int_{d_{n, j}^{\prime}}^{\infty} S_{X}(x) d x=B$. If vectors $\left(\boldsymbol{c}, \boldsymbol{d}^{\prime}\right)$ are constructed by only replacing $d_{n, j}$ in $(\boldsymbol{c}, \boldsymbol{d})$ with $d_{n, j}^{\prime}$, then $\left(\boldsymbol{c}, \boldsymbol{d}^{\prime}\right) \in U$. Furthermore, since $\kappa(x)$ is increasing on $\left(\delta_{\rho}, \infty\right)$ according to part (a) of Lemma B.1, we have

$$
\operatorname{VaR}_{\alpha}(\boldsymbol{c}, \boldsymbol{d}) \geqslant \operatorname{VaR}_{\alpha}\left(\boldsymbol{c}, \boldsymbol{d}^{\prime}\right),
$$

which in turn leads to (B.10). Together with (B.9), we prove (B.6).

For the second part of the proof of establishing (B.8), we again use the same technique as above by demonstrating the following two inequalities:

$$
\begin{gathered}
\operatorname{VaR}_{\alpha}^{*}(U) \leqslant \operatorname{VaR}_{\alpha}^{*}(V) \\
\operatorname{VaR}_{\alpha}^{*}(U) \geqslant \operatorname{VaR}_{\alpha}^{*}(V) .
\end{gathered}
$$

To justify the inequality (B.14), we first note that every coefficient pair $(c, d) \in V$ is a special case of the vectors $(\boldsymbol{c}, \boldsymbol{d})$ in $T_{n}^{\geqslant}$. This can be seen by setting $c_{n, j}=(c) /(n)$ and $d_{n, j}=d, j=1,2, \ldots, n$. Note that the optimization problem in $V$ becomes a two-dimensional problem, instead of $2 n$ dimension as in the general case. Together with (B.6), we have the inequality (B.14).

To verify inequality (15), we proceed as follows. For any vectors $(\boldsymbol{c}, \boldsymbol{d}) \in U$, let us denote $c_{n}:=\sum_{j=1}^{n} c_{n, j}$ and $d_{n}:=\sum_{j=1}^{n}\left(c_{n, j}\right) /\left(c_{n}\right) d_{n, j}$. By treating $\int_{d}^{\infty} S_{X}(x) d x$ as a function of $d$, the convexity property ensures that

$$
B=\sum_{j=1}^{n} c_{n, j} \int_{d_{n, j}}^{\infty} S_{X}(x) d x \geqslant c_{n} \int_{d_{n}}^{\infty} S_{X}(x) d x .
$$

Consequently there exists a coefficient pair $\left(c_{n}, d_{n}^{\prime}\right)$ satisfying $\delta_{\rho} \leqslant d_{n}^{\prime} \leqslant d_{n}$ and $c_{n} \int_{d_{n}^{\prime}}^{\infty} S_{X}(x) d x=B$. As $U \subset T_{n}^{\geqslant} \subset T_{n}^{n} \subset D_{n}^{n}$, we see that $d_{n, j} \leqslant \delta_{\alpha}$ for $j=1,2, \ldots, n$, 
which together with the definition of $d_{n}$, lead to $d_{n} \leqslant \delta_{\alpha}$. Hence $\left(c_{n}, d_{n}^{\prime}\right) \in V$. Moreover,

$$
\begin{aligned}
\operatorname{VaR}_{\alpha}(\boldsymbol{c}, \boldsymbol{d}) & =\delta_{\alpha}+\sum_{j=1}^{n} c_{n, j}\left[d_{n, j}+(1+\theta) \int_{d_{n, j}}^{\infty} S_{X}(x) d x-\delta_{\alpha}\right] \\
& =\delta_{\alpha}+(1+\theta) B+\sum_{j=1}^{n} c_{n, j} d_{n, j}-\sum_{j=1}^{n} c_{n, j} \delta_{\alpha} \\
& =\delta_{\alpha}+(1+\theta) c_{n} \int_{d_{n}^{\prime \prime}}^{\infty} S_{X}(x) d x+c_{n} \cdot d_{n}-c_{n} \cdot \delta_{\alpha} \\
& \geqslant \delta_{\alpha}+(1+\theta) c_{n} \int_{d_{n}^{\prime \prime}}^{\infty} S_{X}(x) d x+c_{n} \cdot d_{n}^{\prime}-c_{n} \cdot \delta_{\alpha} \\
& =\delta_{\alpha}+c_{n} \kappa\left(d_{n}^{\prime}\right) \\
& =\operatorname{VaR}_{\alpha}\left(c_{n}, d_{n}^{\prime}\right),
\end{aligned}
$$

Since $(\boldsymbol{c}, \boldsymbol{d})$ are arbitrary vectors from $U$, inequality (B.15) follows immediately from (B.16) and this completes the proof.

Lemma B.4 If $\alpha<\rho, \kappa\left(\delta_{\rho}\right)<0$ and $\beta\left(\delta_{\rho}\right) \leqslant 1$, then the following inequality is true:

$$
\operatorname{VaR}_{\alpha}^{*}\left(T_{n}^{\leqslant}\right) \geqslant \operatorname{VaR}_{\alpha}^{*}(V) .
$$

Proof For any vectors $(\boldsymbol{c}, \boldsymbol{d}) \in T_{n}^{\leqslant}$, it follows from (A.10) and part (a) of Lemma B.1 that

$$
\begin{aligned}
\operatorname{VaR}_{\alpha}(\boldsymbol{c}, \boldsymbol{d}) & =\delta_{\alpha}+\sum_{j=1}^{n} c_{n, j} \kappa\left(d_{n, j}\right) \\
& \geqslant \delta_{\alpha}+\sum_{j=1}^{n} c_{n, j} \cdot \kappa\left(\delta_{\rho}\right) \\
& \geqslant \delta_{\alpha}+\beta\left(\delta_{\rho}\right) \cdot \kappa\left(\delta_{\rho}\right) \\
& =\operatorname{VaR}_{\alpha}\left(\beta\left(\delta_{\rho}\right), \delta_{\rho}\right) .
\end{aligned}
$$

It is easy to see that $\left(\beta\left(\delta_{\rho}\right), \delta_{\rho}\right) \in V$, which, together with (B.18), implies inequality (B.17), as required. 
140

Lemma $B .5$ If $\alpha<\rho, \kappa\left(\delta_{\rho}\right)<0$ and $\beta\left(\delta_{\rho}\right) \leqslant 1$, then we have $\operatorname{VaR}_{\alpha}^{*}\left(S_{n}^{n}\right)=$ $\operatorname{VaR}_{\alpha}^{*}(V)$, that is Eq. (A.14) holds.

Proof The required relation follows immediately from Lemmas B.2, B.3, B.4 and the partitioning that $T_{n}^{n}=T_{n}^{\geqslant} \bigcup T_{n}^{\leqslant}$.

Lemma B.6 For any $\mu \in \mathcal{M}^{+}$, there exists a sequence of measures $\left\{\mu_{n}, n=1\right.$, $2, \ldots\}$ in $\mathcal{M}_{n}^{+}$such that $h_{n}(x)$ converges pointwisely to $f(x)$ from below, where $h_{n}(x)=\int(x-t)_{+} d \mu_{n}$ and $f(x)=\int(x-t)_{+} d \mu$ for $x \geqslant 0$.

Proof The proof is trivial when the measure $\mu$ is such that $f(x) \equiv 0$. Next, we suppose $f(x) \equiv 0$ does not hold. It is well known that for any nonnegative increasing convex function $f$ defined on $(0, \infty)$, there exists a sequence of nonnegative functions $\left\{h_{n}, n=1,2, \ldots\right\}$ defined on $(0, \infty)$ such that $h_{n}(x)=$ $\sum_{j=1}^{n} c_{n, j}\left(x-d_{n, j}\right)+$ for some constants $c_{n, j} \geqslant 0$ and $d_{n, j} \geqslant 0$ and $\lim _{n \rightarrow \infty} h_{n}(x)=f(x)$ from below for any $x \geqslant 0$. This implies

$$
h_{n}(x) \leqslant f(x) \text { for all } x \geqslant 0 \text { and } n=1,2, \ldots .
$$

See, for example, the proof to Case 1 of Theorem 1.5.7 of Müller and Stoyan (2002, p. 18).

By the definition of $\mathcal{M}^{+}$, for any $\mu \in \mathcal{M}^{+}$, we have $0 \leqslant f(x)=\int(x-t)_{+}$ $d \mu \leqslant x$, which together with (B.19), imply that for any $x>0$ and $n=1,2, \ldots$,

$$
0 \leqslant \frac{h_{n}(x)}{x}=\sum_{j=1}^{n} c_{n, j} \frac{\left(x-d_{n, j}\right)_{+}}{x} \leqslant 1 .
$$

Consequently, by letting $x \rightarrow \infty$ in (B.20) we have $0 \leqslant \sum_{j=1}^{n} c_{n, j} \leqslant 1$ for all $n=1,2, \ldots$. Thus, the sequence of the measures $\left\{\mu_{n}, n \geqslant n_{0}\right\}$ of the form (A.1) with coefficients $c_{n, j}, d_{n, j}, j=1,2, \ldots n$ satisfies the requirements of the lemma and hence the proof is complete. 